\title{
Current Venues of Care and Related Costs for the Chronically Critically Ill
}

\author{
Michael P Donahoe MD
}

\author{
Introduction \\ PMV Patient Definitions From a Cost Perspective \\ Costs and Venues of Care for the PMV Population \\ Acute Care Hospital Costs \\ Acute Care Hospital Payments \\ Acute Care Hospital Step-Down or Special Care Units \\ Post Acute Care Venues for PMV Patients \\ Long-Term Acute Care \\ Skilled Nursing Facilities \\ Episodes of Care \\ Cost Effectiveness \\ Summary
}

\begin{abstract}
The chronically critically ill (CCI) patient population is characterized by a prolonged need for high cost medical interventions, a high 1-year mortality rate, and a very high demand for post acute care services. The best characterized CCI patient population is patients on prolonged mechanical ventilation (PMV). This review will focus on the current knowledge of costs and care venues for the care of this patient population. The PMV population suffers from a prolonged length of acute care hospital stay, median hospital costs 3-4 times the cost of short-term ventilator patients, frequent care venue changes during the course of illness, a small likelihood of discharge to the home environment, yet a hospital mortality that does not differ significantly from the short-term ventilated patient group. The PMV population is projected to double in size by the year 2020. Given the dramatic comparative acute care cost burden of PMV patients, the societal implications for managing both the care burden and the costs of care are staggering. Strategies to improve the efficiency in healthcare for this patient population will be essential. Limitations to the existing care models in the United States will be identified with a focus on our current research deficiencies, which limit healthcare providers and administrators in providing patient focused care for this patient population. Key words: chronically critically ill; prolonged mechanical ventilation; long-term acute care; step-down units. [Respir Care 2012;57(6):867-886. (C) 2012 Daedalus Enterprises]
\end{abstract}

\section{Introduction}

The focus of critical care has traditionally been on stabilization of acute organ system dysfunction and short-

Dr Donahoe is affiliated with the Division of Pulmonary, Allergy, Critical Care Medicine, University of Pittsburgh School of Medicine, Pittsburgh, Pennsylvania. term patient survival. Traditional measures of "success" in critical care intervention trials have focused on weaning or mortality based in the ICU, hospital, or short-term inter-

\footnotetext{
Dr Donahoe presented a version of this paper at the 49th RESPIRATORY CARE Journal Conference, "The Chronically Critically Ill Patient," held September 9-10, 2011, in St Petersburg, Florida.
} 
vals (28-60 d). ${ }^{1-5}$ Advances in critical care interventions have led to improvements in these short-term outcome parameters for patients with certain types of critical illness. ${ }^{6,7}$ With the improvements in short-term mortality for patients with critical illness, the focus has shifted to longer term measures of successful clinical outcome. Survival from critical illness is recognized to be associated with physical, neurocognitive, organ system, and mental health dysfunction in survivors of the "acute" illness. A small, but substantial population of critically ill patients survive the initial critical illness, only to suffer prolonged dependence on life support or the need for long-term therapeutic interventions. These patients have been grouped under the classification of "chronically critically ill" (CCI) patients. In general, the CCI patient population is characterized by heterogeneity, which resists simple categorization, a prolonged and poorly predicted need for high cost interventions, a high longer term ( $\sim 1$ year) mortality rate, and a very high demand for post acute care (PAC) services. ${ }^{8,9}$ The best characterized component of the CCI population is patients on prolonged mechanical ventilation (PMV). This review will focus on the venues of care for the CCI with a focus on the PMV population and our current knowledge of their associated costs of care, and highlight gaps in our knowledge base which limit healthcare providers and administrators in providing patient focused care for this patient population.

\section{PMV Patient Definitions From a Cost Perspective}

An assessment of CCI patients and their "post-acute" management is complicated by the precision of the CCI definition. Most investigations of the CCI population have focused on the subsegment of patients who require either ICU care or some duration of mechanical ventilation (MV). These analyses have often involved the mining of large data sets, typically Medicare cost and payment files. ${ }^{10-12}$ Although most ICU physicians can easily identify a CCI patient, characterization for the purposes of research investigation and data mining for comparative cost analysis usually requires some compromise. Readily identifiable charge parameters include the need for MV (ventilator charge) or a long-term ICU stay (ICU bed charge). The availability of ventilator charges has allowed isolation of patients who require PMV. Yet even the definition in this

\section{The author has disclosed no conflicts of interest.}

Correspondence: Michael P Donahoe MD, Division of Pulmonary, A1lergy, Critical Care Medicine, University of Pittsburgh School of Medicine, Pittsburgh PA 15213. E-mail: donahoem@upmc.edu.

DOI: $10.4187 /$ respcare. 01656
Table 1. Comparison of Resource Requirements of Patients Who Require Mechanical Ventilation for $>48$ Hours, Classified by Different Methodologies

\begin{tabular}{|c|c|c|c|}
\hline Parameter & $\begin{array}{l}\text { Short Term } \\
\text { Ventilation } \\
(n=524)\end{array}$ & $\begin{array}{l}\text { DRG } 541 / 542 \\
\quad(n=267)\end{array}$ & $\begin{array}{c}\geq 21 \text { Days } \\
(n=114)\end{array}$ \\
\hline Hospital mortality, \% & 43 & $20^{*}$ & $31 \dagger$ \\
\hline 1 year mortality, $\%$ & 59 & $48^{*}$ & $58 \dagger$ \\
\hline ICU stay, mean (range) d & $8(5-12)$ & $22(14-31)^{*}$ & $30(24-41) \dagger$ \\
\hline Hospital stay, mean (range) d & $15(9-21)$ & $29(22-28)^{*}$ & $39(30-52) \dagger$ \\
\hline $\begin{array}{l}\text { Hospital costs per } 1 \text { year } \\
\text { survivor }\end{array}$ & $\$ 165,075$ & $\$ 266,105^{*}$ & $\$ 423,596 \dagger$ \\
\hline Ineffective care, $\%$ & 10 & 22 & 47 \\
\hline \multicolumn{4}{|l|}{$\begin{array}{l}\text { Discharge disposition, } \\
\text { no. }(\%)\end{array}$} \\
\hline Home & $90(17)$ & $19(7)$ & $5(4)$ \\
\hline Ventilator facility & $0(0)$ & $45(17)$ & $9(8)$ \\
\hline Skilled nursing facility & $81(15)$ & $60(22)$ & $28(25)$ \\
\hline Rehabilitation & $111(21)$ & $77(29)$ & $27(24)$ \\
\hline Other Hospital & $15(4)$ & $13(5)$ & $9(8)$ \\
\hline \multicolumn{4}{|c|}{ 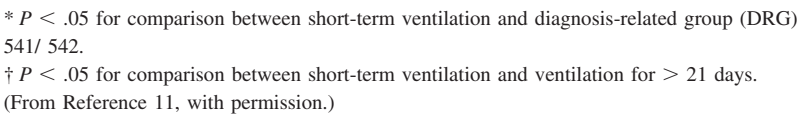 } \\
\hline
\end{tabular}

theoretically "homogeneous" segment of the CCI population can describe different clinical behaviors and resource requirements. ${ }^{11} \mathrm{~A}$ consensus group defined PMV as ventilation for $\geq 21$ days, based upon the observation that the majority of patients transferred to long-term care units on MV have received ventilation for at least 21 days..$^{13-15}$ In contrast, other investigators have favored Medicare's definition of MV for $>96$ hours, with tracheostomy as the marker for PMV (diagnosis-related groups [DRGs] $541 / 542$, formerly 483). ${ }^{16}$ In a post hoc analysis of a prospectively observed population of patients requiring MV for $>48$ hours, Cox et al compared the outcome and resource utilization in 2 distinctly defined CCI populations. A cohort of hospital patients requiring acute MV for $>48$ hours were divided into 3 study groups consisting of: patients who underwent tracheostomy for non-head and -neck diagnoses (DRG 541/542); patients who experienced PMV for $\geq 21$ days; and patients ventilated between 2 and 21 days and who did not receive a tracheostomy (shortterm ventilation). ${ }^{11}$ As illustrated in Table 1 , PMV patients defined by DRG 541/542 had significantly longer ICU and hospital stays, and their hospital costs were substantially higher than the short-term ventilation cohort. Ineffective care, defined as a combination of high resource consumption ( $>\$ 100,000$ per hospitalization) and early death $(<100 \mathrm{~d}$ survival), was greatest in the population characterized by $>21$ days on MV. Resource requirements were also highest for the $>21$ day MV population. Potentially ineffective care was associated in this group with 
age, days of ventilation, male sex, and the number of pre-admission activities of daily living. Ineffective care was not associated with day 1 acute physiology score, admission source, or admitting service.

The 2 most commonly proposed definitions for PMV appear to provide comparable baseline clinical characteristics and trends in survival, disposition, and resource utilization. However, PMV defined as ventilation for $>21$ days more specifically characterizes patients who are outliers in resource consumption and at risk for potentially ineffective hospital care. The definitions also identify a patient population characterized by a high mortality subsequent to the index hospitalization discharge, almost doubling in the subsequent year for both PMV definitions. This high subsequent year mortality for surviving patients from prolonged critical illness has also been confirmed in additional cohorts. ${ }^{17}$ Patients in all 3 categories of MV have a very high need for PAC facilities with less than $20 \%$ of patients being discharged to home in all categories.

A high post acute ICU care mortality is not uniformly reported, however. Laupland et al studied the long-term outcome of all adult ( $\geq 18$ years old) admissions to the multisystem and cardiovascular surgical ICUs in the Calgary Health Region from July 1, 1999, to March 31, $2002 .{ }^{18}$ A prolonged ICU admission was defined as $\geq 14$ days, independent of the need for MV. During the study, 216 of the 4,845 patient admissions $(4 \%)$ had a prolonged ( $>14 \mathrm{~d}$ ) admission to the ICU. A higher severity of illness, the presence of shock, and bloodstream infection were independently associated with a prolonged ICU admission, and cardiovascular surgery was associated with a lower risk. Patients with prolonged ICU admissions were nearly twice as likely to die as patients with shorter ICU admissions. However, among the 3,924 survivors to hospital discharge, the rates of mortality during the year following ICU admission were not different between patients admitted for $<2$ days and those admitted for $>14$ days.

Approximately $50 \%$ of patients in this prolonged surgical ICU cohort required MV. These studies raise important questions as to whether the high post discharge mortality reported in PMV patients is a function of the severity of illness or may possibly reflect variations in post discharge acute care.

\section{Costs and Venues of Care for the PMV Population}

\section{Acute Care Hospital Costs}

The hospitalization costs of patients who require PMV have been described by Zilberberg et al, using direct comparison of outcome and cost data for short and long-term MV patients in the acute care setting. ${ }^{19}$ Using calendar year 2003 data from the National In-Patient Survey, they examined the outcomes and costs of adult patients
( $>18$ years old) in approximately 1,000 United States in-patient facilities. Patients were grouped by administrative coding into a population on MV for $<96$ hours and those on PMV ( $\geq 96 \mathrm{~h}$ ). Over 50\% of PMV patients originated from urban teaching hospitals. The PMV patients were characterized by a higher prevalence of coexisting conditions than MV $<96 \mathrm{~h}$ patients. The prevalence of both sepsis and ARDS was higher in the PMV population. Although the burden of illness was higher in the PMV group compared with the MV $<96$ h group, by any illness severity measure examined, as was their predicted hospital mortality, the actual in-hospital mortality rates were similar between the 2 populations. Resource utilization differed greatly between the groups. Median hospital costs for MV patients were $\$ 13,434$ (interquartile range $\$ 7,420$ to $\$ 24,194$ ) and for PMV $\$ 40,903$ (interquartile range $\$ 24,905$ to $\$ 68,865$ ). Notably, average costs per day were similar between the groups $(\$ 2,666$ for PMV and $\$ 3,228$ for MV). Although PMV subjects comprised only $39 \%$ of all persons undergoing MV, they accounted for $64 \%$ of all annual in-patient costs. Transfers to non-acute care centers, such as a skilled nursing facility (SNF), were more likely in the PMV group than in the MV $<96 \mathrm{~h}$ group (36\% vs $20 \%$, respectively), while a routine discharge home was one half as likely in the PMV group (15\% PMV vs 30\% MV). Conversely, proportions of discharges home with home healthcare, transfers to shortterm hospitals (ie, acute-care facility), and in-hospital mortality were similar for the PMV and MV groups (Fig. 1).

PMV patients in this series were characterized by a higher burden of chronic illness, a markedly prolonged stay, triple the median hospital costs of the MV $<96 \mathrm{~h}$ patients, a relative 50\% decrease in the likelihood of a routine discharge home, yet a hospital mortality for the PMV group that did not differ from that in the MV $<96 \mathrm{~h}$ group (35\% vs $34 \%$, respectively). The latter result was divergent from the mortality rates predicted by the database indicators, where substantially higher hospital mortality was predicted for the PMV than for the MV group. These data are consistent with other studies of mortality prediction models, which suggest that hospital mortality in PMV patients is not determined by traditional parameters. ${ }^{20}$ The cohort was not examined for longer term mortality post discharge, nor did they consider the role of discharge venue in acute care hospital costs or total episode of illness costs.

Based upon additional analysis from the National InPatient Survey data set (2000-2005), these investigators have projected a historic annualized increase in PMV patients of $\sim 5.5 \%$ per annum. ${ }^{21}$ The fastest annualized growth $(7.9 \%)$ was observed in the 44-65 year age group. Factoring in both age-specific growth in PMV population and overall United States adult population changes, they projected PMV patients to more than double, from approxi- 

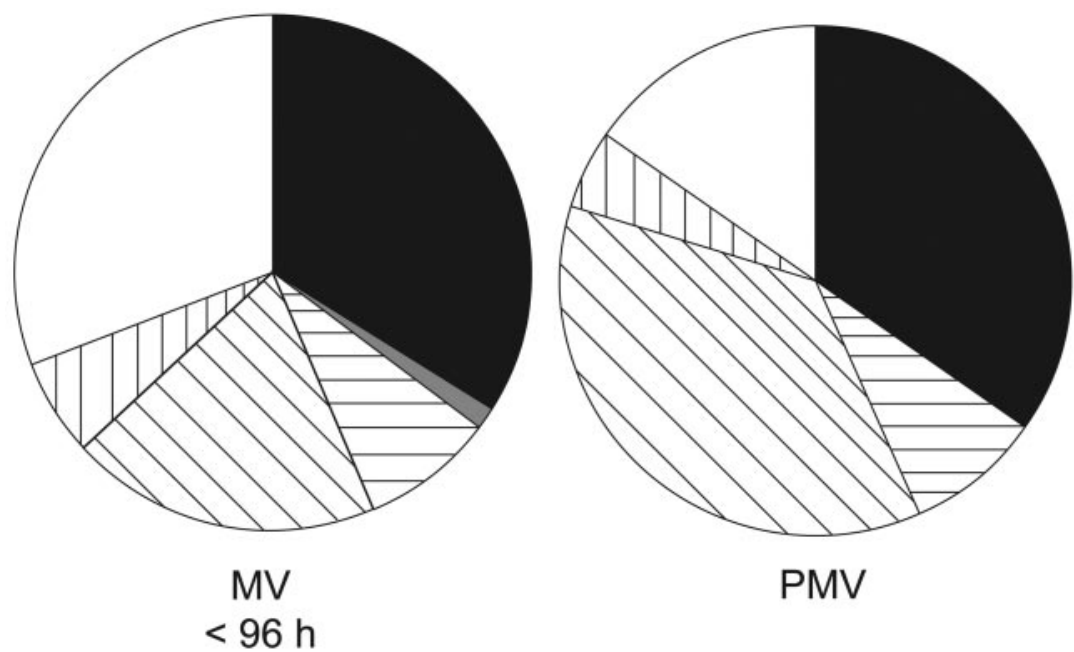

Died in hospital

Against medical advice/alive, destination unknown

Home health care

Transfer: skilled nursing facility, intermediate care, other type

Transfer: short-term hospital

Routine

Fig. 1. Discharge destination by type for adult hospital discharges with mechanical ventilation for $<96$ hours (MV $<96 \mathrm{~h})$ and those with prolonged acute ( $\geq 96 \mathrm{~h}$ ) mechanical ventilation (PMV). (Adapted from Reference 19.)

mately 250,000 cases in 2000 to 605,898 cases by the year 2020. Given the dramatic comparative acute care cost burden of PMV patients, the societal implications for managing both the care burden and the costs of care are staggering. Strategies to improve the efficiency in healthcare for this patient population will be essential.

Dasta and colleagues considered the relative daily costs of ICU patients and the role of MV in that cost analysis. ${ }^{12}$ These investigators analyzed the daily cost of ICU care in 51,009 patients in relation to the need for MV from an administrative database in calendar year 2002. ICU patients requiring MV had a significantly higher crude mortality rate than non-MV ICU patients $(23.7 \%$ vs $5.9 \%)$, and were more likely to transfer to PAC facilities. MV patients were ventilated a mean of 5.6 days and had significantly more ICU days than non-MV patients $(6.9 \mathrm{~d}$ vs 2.9 d). MV patients accrued significantly more cost during their ICU $(\$ 31,574$ vs $\$ 12,931)$ and hospital stay $(\$ 47,158 \pm \$ 57,703$ vs $\$ 23,707 \pm \$ 34,545)$. Hospital days were significantly greater for the MV patients $(14.4 \pm 16.1 \mathrm{~d}$ for MV vs $8.5 \pm 10.7 \mathrm{~d}$ for patients not requiring MV). Mean daily ICU costs were greatest on the first day of ICU treatment $(\$ 7,728 \pm \$ 8,509)$, decreased on day 2 $(\$ 3,872 \pm \$ 4,223)$, and became stable from day 3 forward $(\$ 3,436 \pm \$ 3,550)$. The greatest day 1 cost occurred in surgical patients (mean $\$ 9,165 \pm \$ 9,438$ ), followed by the trauma ICU cohort (mean $\$ 8,199 \pm \$ 7,880$ ). By day 3 , daily costs were stable in all cohorts, approximately $\$ 3,500 / \mathrm{d}$ in the surgical and trauma ICU cohorts and approximately $\$ 3,000 / \mathrm{d}$ in the medical ICU cohort. MV was the greatest independent predictor of $\operatorname{cost}(P<.001)$ providing an incremental ICU cost of $\$ 1,522 /$ patient/d. Daily costs were consistently greater for patients requiring MV, and the incremental cost was greatest on ICU day 1 and significantly greater in surgical than medical patients.
In this series, MV patients were in the ICU an extra 4 days and in the hospital an extra 6 days in total, compared with patients who did not receive MV. The consistent costs for MV patients beyond hospital day 3 suggest that the early hospital days are characterized by variable costs related to surgical intervention, pharmaceuticals, or diagnostic investigation. The latter days of ICU MV may be characterized by more fixed costs related to the ICU bed, nursing, and respiratory therapy support.

A stabilization of ICU costs following the admission days has been independently confirmed by Rapoport and colleagues. ${ }^{22}$ Data on consecutive medical-surgical ICU patients from 2 Massachusetts hospitals (Baystate Medical Center and New England Medical Center) during January to September of 1998 were explored, and the cost for each day of the ICU stay and post-ICU hospital stay was calculated. As illustrated in Table 2, mean and median ICU costs are greatest on ICU day 1 and appear to stabilize on subsequent hospital days. The initial day ICU costs appear to be 4-fold greater than comparable non-ICU costs, but this ratio reduces to $\sim 2.5$ times post-ICU days after the initial day, and remains constant over the remaining illness duration.

The relationship between ventilator days and hospital costs is slightly more complex when we attempt to compare venues of care within the acute care environment. This introduces the concept of "cost savings" and requires a greater exploration of the type of costs reported in these investigations. Kahn and colleagues conducted a retrospective cohort study of patients undergoing MV for $>48$ hours in the ICUs at the University of Pennsylvania during fiscal year 2006. ${ }^{23}$ In their analysis, 3 types of hospital costs were considered. Total costs included the costs for all services for each patient day. Variable costs were defined as total costs excluding overhead costs, 
Table 2. Summary of Daily ICU Costs, Compared to Non-ICU Costs, by Hospital Stay for Baystate Medical Center

\begin{tabular}{|c|c|c|c|c|c|}
\hline $\begin{array}{l}\text { ICU } \\
\text { Day }\end{array}$ & $N$ & $\begin{array}{c}\text { Mean } \\
\text { Costs } \\
\text { per } \\
\text { Patient } \\
(\$)\end{array}$ & $\begin{array}{c}\text { Median } \\
\text { Costs } \\
\text { per } \\
\text { Patient } \\
(\$)\end{array}$ & $\begin{array}{l}\text { Ratio of } \\
\text { Mean ICU } \\
\text { Costs to } \\
\text { Post ICU } \\
\text { Mean } \\
\text { Costs }\end{array}$ & $\begin{array}{c}\text { Ratio of } \\
\text { Median ICU } \\
\text { Costs to } \\
\text { Post ICU } \\
\text { Median } \\
\text { Costs }\end{array}$ \\
\hline 1 & 385 & 4,234 & 3,052 & 4.6 & 3.7 \\
\hline 2 & 272 & 2,573 & 2,550 & 2.8 & 2.7 \\
\hline 3 & 196 & 2,475 & 2,092 & 2.7 & 2.5 \\
\hline 4 & 157 & 2,531 & 2,124 & 2.8 & 2.6 \\
\hline 5 & 133 & 2,470 & 2,094 & 2.7 & 2.5 \\
\hline 6 & 113 & 2,307 & 2,053 & 2.5 & 2.5 \\
\hline 7 & 92 & 2,359 & 2,135 & 2.6 & 2.6 \\
\hline 8 & 81 & 2,417 & 2,115 & 2.6 & 2.6 \\
\hline All ICU days $>8$ & 73 & 2,226 & 2,020 & 2.4 & 2.4 \\
\hline Post ICU days & 363 & 919 & 825 & & \\
\hline
\end{tabular}

but including staff salaries and equipment costs. Directvariable costs were defined as variable costs, excluding staff salary and equipment costs. Direct-variable costs are the supply costs for providing a specific service and, therefore, represent the actual costs savings that could be achieved by not providing that service.

The average total cost for each patient was $\$ 69,472$, of which $\$ 12,773$ were direct-variable costs $(18.4 \%)$. The average ICU cost for each patient was $\$ 54,468$, of which $\$ 10,516$ were direct-variable costs $(19.3 \%)$. Directvariable costs were highest for the blood bank $(43.5 \%)$ and pharmacy (48.2\%), and relatively low for radiology (8.2\%) and respiratory therapy $(2.9 \%)$. The total, variable, and direct-variable costs for each ICU day are shown in Figure 2. Costs were highest during the first 2 days of intensive care and decreased significantly in subsequent ICU days. The average direct-variable costs of an ICU day was $\$ 1,751$. The difference in direct-variable costs between the last ICU day and the first ward day was only $\$ 118$ (95\% CI \$21-\$190) for patients who remained on MV for $>3$ days. The same pattern was seen examining the cost difference between the last ventilator day and the first non-ventilator day, where the direct-variable cost difference was only \$106 (95\% CI \$76-\$156).

Kahn et al's analysis illustrates that comparative venue hospital cost analyses are complex and that many of our current estimates have significant methodological limitations. The majority of hospital costs are fixed costs, representing the overhead of running a hospital, and are not impacted by reducing stay in a specific area of the acute care hospitalization. Reducing ventilator or ICU days does not impact the fixed costs. Reducing ICU or ventilator days does not save the cost of the ICU day, but rather the

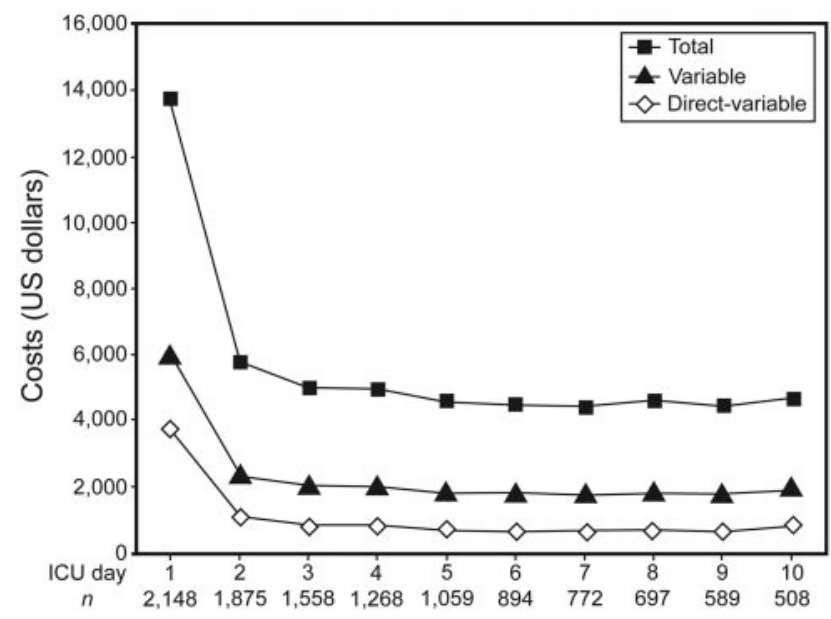

Fig. 2. Mean total costs, variable costs, and direct-variable costs for each ICU admission in a cohort of mechanically ventilated patients at the University of Pennsylvania. (From Reference 23, with permission.)

marginal cost of the ICU day compared to the floor or step-down day.

In the acute care setting, both MV and PMV patients are characterized by high resource consumption. As a high cost population, PMV patients are a focus for hospital managers to control daily expenditures and consider venues of care other than the ICU. However, cost control strategies should proceed in an informative manner and typically suffer from the lack of detailed cost analysis in current intervention trials. ICU cost control strategies that utilize alternative venues but result in extension of the hospital stay could produce unfavorable results in patient costs for the entire hospital course. This places great emphasis on understanding the impact of specific hospital based intervention strategies for MV patients (ie, weaning and mobility protocols) on the cost implications for care delivery. Many current clinical trials use ICU or ventilator days as surrogates for cost information. Alternatively, average daily costs are compared between venues of care, assigning a single unit cost to an ICU or step-down day without recognizing the substantial impact of day $1-2$ costs on the mean ICU patient cost. Intervention trials should attempt to measure hospital costs directly when possible with a focus on marginal variable costs rather than total average costs.

Age appears to be another important variable that influences hospital costs in patients on MV. In a cohort of 817 patients requiring MV for $>48$ hours, Chelluri et al examined the influence of age on hospital costs. ${ }^{24}$ The highest proportion of total hospital costs in this cohort was attributed to the ICU room cost, which ranged from $7.1 \%$ to $80.2 \%$ of the total hospital costs across patients. Similar to prior investigations, patients on MV in the medical ICU were less costly than those managed in the surgical ICU 
Current Venues of Care and Related Costs for the Chronically Critically Ill

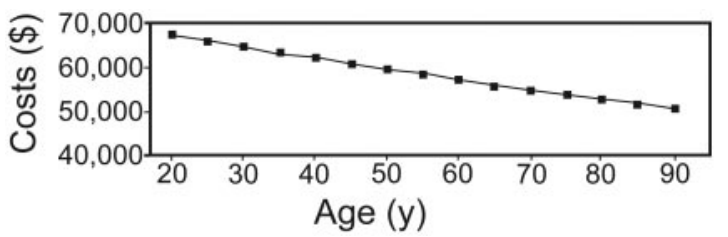

Fig. 3. Expected total hospital costs by age group in a cohort of patients on mechanical ventilation for $\geq 48$ hours. Based on multiple regression models, costs are for a female, surgical intensive care unit patient, with other than managed care insurance, who did not change resuscitation orders to less treatment, who survived the hospitalization, who had a Charlson comorbidity score of 2.2, a hospital stay of 23.5 days, and an Acute Physiology Scale score on day 1 of 56.0. (From Reference 24, with permission.)

(median total costs $\$ 41,031$ vs $\$ 63,438$, median daily costs $\$ 2,308$ vs $\$ 2,954)$. Median total costs (and daily cost) for hospital survivors and nonsurvivors were $\$ 59,966(\$ 2,562)$ and $\$ 43,066(\$ 3,016)$, respectively. In this cohort, age was statistically associated with total hospital, pharmacy, radiology, laboratory, and miscellaneous costs, with older patients having lower overall costs. Restricting analysis to patients who had costs in their respective departments, ICU room and respiratory care costs did not differ with the age of the patients, however. Using multivariate analyses, the authors defined expected total hospital costs for a given age group, attempting to match for severity of illness, insurance status, resuscitation preference, and hospital stay. For total hospital and most of the departmental costs, including operating room/anesthesia, blood bank, pharmacy, radiology, laboratory, and miscellaneous costs, older subjects had significantly lower expected costs than younger persons, even after adjusting for potential confounders such as sex, severity of illness, and stay. For respiratory care, age was positively associated with costs. There was no significant relationship between age and either floor or ICU costs. Comparative analysis of single day costs (day 2 of ICU hospitalization) continued to demonstrate a significant influence of age on resource consumption, suggesting that more frequent limitation of care is not the sole explanation for the cost reduction in older patients. The relationship between age and costs appeared to be linear, suggesting that the decrease in costs is present across all age groups (Fig. 3).

This investigation raises many questions about the influence of age on ancillary costs. The implication is that patients and/or providers are influenced by undefined bias in their selection of care based upon the patient's age for all parameters except respiratory care. This pattern for resource utilization suggests cost reductions in hospital patients may need to consider age as an important variable and not neglect the role of inappropriate treatments in younger patients.

\section{Acute Care Hospital Payments}

Given that PMV patients have high costs influenced by variables such as ICU location, hospital day, and age, how does hospital reimbursement for these patients compare to their significant costs. Do these patients represent a significant financial gain or loss to the acute care hospital?

For the acute care environment, payment is established by the payer, but the majority of payers follow the guidelines established by Medicare. The need for alternative venues for PMV patients was heightened in 1983, when Medicare (and subsequently all payers) shifted from a cost based payment model to a prospective payment system (PPS). The Medicare PPS relies upon classification of each patient into a specific DRG upon discharge. The DRG payment to the hospital is established by multiplying the average payment for the provider type (the base rate) by the relative resource consumption of that DRG (the DRG weight). The calculated payment is then subjected to a number of modifying factors that attempt to incorporate additional reimbursement for hospitals with commitments to medical education (the indirect medical education adjustment), a high portion of uncompensated care (the disproportionate share adjustment), as well as geographic adjustments for capital costs and labor costs (the wage rate index adjustment). The PPS includes an outlier policy with additional payments for patients who are clearly more costly than the average patient expected by the DRG reimbursement. The intention of PPS is to pay all hospitals the same for similar patients, with the assumption that variability in patient severity will balance out for a given DRG. In the PPS model, the provider (hospital) is incentivized to reduce the cost of care for a given hospitalization to achieve a greater operating margin. PPS provides a substantial financial disincentive for providers to treat high-cost cases such as patients with PMV. In the short-term acute care (STAC) hospital DRG system, when costs exceed the DRG reimbursement, the provider must absorb a non-reimbursed "fixed-loss outlier threshold," after which Medicare will reimburse the provider $80 \%$ of a formula-derived "cost." In keeping with the cost-control premise for PPS, the outlier policy is intended to provide a financial loss to the hospital.

Although PMV patients qualify for some of the highest paying DRGs, their long hospital stays and high resource demands dictate that they are frequently high cost outliers, whose costs far exceed the expected payment for services delivered. ${ }^{25}$ The change to the PPS payment model, as well as the need to create additional ICU bed capacity, quickly prompted acute care hospitals to seek alternative care venues for the PMV patient, outside the ICU environment. ${ }^{26}$

The acute care environment is focused on cost control via one of 2 pathways. Reduction in variable costs for the 
daily care of patients on PMV is one avenue to improve the efficiency of care for the PMV population. Interventions that shorten the hospital stay, in spite of incremental daily costs, may still be very favorable. A second strategy is to shift the venue of care from the high cost burden ICU to step-down or long-term acute care units. Barriers to patient movement from high cost to low cost environments once medical stability is achieved should be carefully investigated with respect to their effects on both patient safety and cost reduction. Reducing the hospital stay of chronically critically ill patients will limit reimbursement deficits that occur when the costs of their acute hospital care exceed their DRG and outlier payments. Acute care hospitals benefit from cost savings in the PMV patient primarily in association with transfers to external acute care venues, as the savings with internal transfers appear to be relatively modest. In addition, acute care hospitals benefit from transition in the venues of care by freeing ICU beds that would otherwise be utilized for weeks or months by a single patient and thus promote patient flow from emergency departments, operating rooms, and acute hospital wards.

\section{Acute Care Hospital Step-Down or Special Care Units}

Numerous reports have documented the role of stepdown or special care units (SCUs) in the care of patients with a need for PMV. ${ }^{27-30}$ Both general medical-surgical SCUs, and disease specific (ie, COPD) units have been described. SCUs provide care for patients following prolonged intervals of ICU MV (mean 13-55 d), with widely variable discharge survival rates (33-96\%), discharge weaning rates (25-96\%), and variable 1-year survival (16$68 \%) .27-31$ The wide variability in outcome measurements is generally attributed to case-mix variability. The small size of the individual series (61-420 patients) and limited details regarding the operational structure limits consideration of how care models may influence patient outcome.

One of the largest case series of patients with PMV managed in an acute hospital specialty unit has been reported by Gracey and colleagues from the Mayo Clinic. ${ }^{27}$ The chronic ventilator dependent SCU accepted ventilator dependent patients with a tracheostomy, based upon their ability to cooperate or potentially to cooperate in a rehabilitation program, with a goal to wean them from MV. Patients in a persistent vegetative state, in need of invasive hemodynamic monitoring, or suffering from hemodynamic instability were excluded. The investigators were able to compare direct and indirect costs over the time interval from 1993 to 1998 for all their intensive care units and the SCU, including allied health salaries, equipment depreciation, minor equipment, dietetics food supply, clinical education, space, equipment rental, equipment main- tenance, and office supplies. Their analysis did not include laboratory, radiology, pharmaceutical, or respiratory therapy costs, using the justification that these variable costs are patient dependent. During the 6 year study interval, 964 patients were admitted to the SCU, including 420 (43\%) newly ventilator dependent patients with tracheostomy, 339 (35\%) patients requiring noninvasive ventilation (NIV) or tracheostomy with intensive respiratory care, and 205 (21\%) non-unit patients admitted from an out-patient status.

Using the assumptions of an equivalent stay in the other acute care hospital ICUs, the investigators calculated a $\$ 4.8$ million savings from the use of the chronic ventilator dependent unit, including $\sim \$ 3.2$ million in the newly ventilator dependent patients. From this population, 64\% of patients were weaned, with a $7 \%$ hospital mortality rate. This cost analysis failed to adjust for the variable cost of ICU days and the specific reduction of direct-variable costs, based upon the venue of care. The investigators also likely overestimated their fixed cost savings, based upon the analysis by Kahn et al previously discussed. ${ }^{23}$

Latriano and colleagues reported their experience with a nonmonitored respiratory care floor (RCF) for 224 mechanically ventilated patients. ${ }^{30}$ All patients were in a hemodynamically stable condition, not requiring vasoactive drug infusions for at least 24 hours prior to transfer. Oxygen requirements were stable and $\leq 50 \%$ on $\leq 10 \mathrm{~cm} \mathrm{H}_{2} \mathrm{O}$ PEEP. Preferred admission criteria included tracheostomy prior to transfer and the ability to interact with the care providers. Patients received a mean of 23 days MV in the ICU prior to admission, and required a mean of 49 days receiving $\mathrm{MV}$ in the RCF. Hospital mortality rate was $50 \%$. Patient discharge status included the acute care ICU (14.3\%), home (31.3\%), SNF (23.9\%), and long-term acute care hospital (LTAC) (6.2\%). The cost analysis suggested that the estimated costs per patient day were $\$ 453$ in the $\mathrm{RCF}$ and $\$ 830$ in the ICU, suggesting a 50\% reduction in costs. The cost reduction was attributed primarily to a reduction in nursing and physician costs, and a smaller reduction in supply costs.

Douglas and colleagues conducted one of the very few randomized clinical trials of PMV patients managed in a traditional ICU setting, compared to an SCU for patients who required MV. ${ }^{31}$ Patients were selected for investigation based upon an ICU stay $>7 \mathrm{~d}$, no vasoactive medications, no hemodynamic monitoring, an Acute Physiology and Chronic Health Evaluation (APACHE II) score $\leq 15$, Therapeutic Intervention Score II or III of 10-39, and inability to be cared for on a regular floor unit. The subjects were randomized to care in the SCU or the traditional ICU. The SCU demonstrated a reduced hospital mortality and cost reductions, compared to the traditional ICU, when considered as cost to produce a survivor (Table 3). The randomized nature of this trial controls for the 
Table 3. Costs in an ICU Versus a Special Care Unit

\begin{tabular}{lcc}
\hline \hline & \multicolumn{2}{c}{ Costs, mean \pm SD } \\
\cline { 2 - 3 } & \multicolumn{1}{c}{ ICU* } & Special Care Unit $\dagger$ \\
\hline Total & $\$ 87,232 \pm \$ 73,111$ & $\$ 74,051 \pm \$ 43,518$ \\
Margin & $-\$ 14,255 \pm \$ 49,344$ & $-\$ 8,491 \pm \$ 37,163$ \\
Standard & $\$ 18,524 \pm \$ 31,352$ & $\$ 13,848 \pm \$ 2,652$ \\
Radiology & $\$ 2,388 \pm \$ 2,306$ & $\$ 1,649 \pm \$ 1,936$ \\
Respiratory therapy & $\$ 3,496 \pm \$ 3,394$ & $\$ 3,427 \pm \$ 3,351$ \\
Pharmacy & $\$ 4,922 \pm \$ 4,133$ & $\$ 4,438 \pm \$ 4,506$ \\
Cost per survivor & $\$ 80,534 \pm \$ 34,908$ & $\$ 59,842 \pm \$ 34,118$ \\
& & \\
$* n=70$ & & \\
$\dagger n=136$ & & \\
(Data from Reference 31.) & & \\
\hline
\end{tabular}

impact of care venue on hospital stay. The careful cost analysis suggests this study may best accurately reflect the true potential cost reductions of a SCU.

While these data support the clinical safety of specialized care units, the analysis of cost/benefit is complicated by the analysis design. Utilization of less resources such as nursing and respiratory care personnel for the provision of patient care in a SCU environment will logically lead to an expected reduction in patient care costs per day. This is confirmed in multiple SCU case series reports. Additionally, alternative medical staff strategies have been examined in SCUs with no important changes in patient outcome, offering the potential for further cost reductions. ${ }^{32}$ Yet the lack of randomization limits conclusions regarding the impact of SCUs on the duration of MV and discharge disposition of the patient. Cost comparisons traditionally "assume" a constant stay between the SCU and ICU environment. This assumption remains generally untested. Any variation in SCU ventilator days or readmission rates to the ICU, in comparison to traditional ICU care, would substantially impact the overall cost of patient care. The overall advantage to hospital costs will also vary based upon the acuity, resource needs, and payment structure of patients supported in the ICU with the opening of bed capacity by the specialized care unit. ${ }^{33}$

Specialized patient care units report variable outcomes for both short-term weaning success and long-term mortality. The traditional explanation is that clinical outcomes are variable based upon variations in case-mix and admission criteria for these environments. Unexplored is the question of whether the associated change in care resources transitioning from acute to step-down care may influence patient outcome.

Polverino and colleagues provided some insight into this question with their reported experience in an Italian specialized respiratory intensive care unit from 1991 to 2005 in 3,106 patients. ${ }^{34}$ They studied 2 patient populations: a group with difficult weaning with tracheostomy after previous ventilation longer than 15 days in the ICU, and a group with acute respiratory failure needing NIV and presenting with acute deterioration in neurological status, severe hypercapnia with respiratory acidosis, and tachypnea. Over the study interval, the number of respiratory care unit beds and total admissions per year increased dramatically (Table 4). Admission comorbidities and prior ICU stay also increased, suggesting a more severely compromised patient population. During the study interval, the investigators also noted a progressive increase in the RICU stay, a decline in the weaning success rate, and a decline in the percentage of patients discharged to home (Table 5). Comparison of physician/patient, nurse/patient, and physiotherapist/patient ratios over time confirmed a reduction in physician/patient ratio, an increase in physiotherapist/ patient ratio, and a constant nurse/patient ratio. The investigators found significant correlations between the doctor/ patient ratio and the rates of weaning success. The investigation raises important questions regarding the relationship between staffing models and patient outcomes. If we consider time on MV and discharge venue as highly important outcome variables for the PMV patient population, then incremental costs that shorten ventilator time or improve discharge to home become less important.

\section{Post Acute Care Venues for PMV Patients}

In addition to acute ICU and hospital based specialized care units, the PMV patient population in the United States is also provided care in a variety of PAC environments. PAC providers include in-patient rehabilitation facilities (IRFs), SNFs, and LTACs. Patients discharged to home may also require the support of home health agencies. LTACs are specifically intended in the United States for the patient population with prolonged acute care needs. PAC utilization in the United States, as is true for healthcare in general, has been driven not only by the clinical needs of the PMV population, but also by the organization of the PAC payment systems. Because Medicare pays the costs for approximately $70 \%$ of all PAC patients, Medicare payment systems have been the main influencing force in the development of the PAC environment. In addition, private insurers often tend to follow Medicare's lead. Payment system incentives in the United States are among the most powerful forces that determine the available care delivery models.

Kahn et al has provided a retrospective cohort analysis of the discharge location of fee-for-service Medicare beneficiaries (age $>65$ ) who required intensive care hospitalization between 1997 and 2006. ${ }^{10}$ In calendar year 2006, $57.7 \%$ of ICU patients were discharged to home, while only $20.6 \%$ of mechanically ventilated patients were discharged to home (Table 6). 
Current Venues of Care and Related Costs for the Chronically Critically Ill

Table 4. Patient Characteristics, Respiratory ICU Occupation, and Staffing of 5 Respiratory ICUs Over a 15 Year Study Interval

\begin{tabular}{|c|c|c|c|c|}
\hline & $1991-1995$ & $1996-2000$ & $2001-2005$ & $P$ \\
\hline Age, y & $78 \pm 1$ & $76 \pm 4$ & $73 \pm 5$ & $<.05$ \\
\hline APACHE II score & $13 \pm 1$ & $13 \pm 1$ & $14 \pm 1$ & $<.05$ \\
\hline Previous ICU stay, $\mathrm{d}$ & $25 \pm 1$ & $28 \pm 2$ & $32 \pm 3^{*}$ & .002 \\
\hline Comorbidities & $1.8 \pm 0.8$ & $2.6 \pm 0.5$ & $3.0 \pm 0.6$ & .050 \\
\hline Admission diagnosis, no. (\%) & & & & $<.001$ \\
\hline COPD & $574(64)$ & $705(72)$ & $789(65)$ & \\
\hline Cardiovascular disease & $206(23)$ & $165(17)$ & $215(18)$ & \\
\hline Neurologic or neuromuscular disease & $126(14)$ & $116(12)$ & $210(17)$ & \\
\hline Total beds & $12 \pm 2$ & $15 \pm 1$ & $24 \pm 6^{*}$ & .001 \\
\hline Total admissions/y & $180 \pm 14$ & $197 \pm 22$ & $244 \pm 45^{*}$ & .02 \\
\hline No. of patients/bed/year & $15 \pm 2$ & $13 \pm 1$ & $10 \pm 1^{*}$ & .002 \\
\hline Nurse/patient ratio & $1: 2.6$ & $1: 2.2$ & $1: 2.4$ & $<.05$ \\
\hline Physiotherapist/patient ratio & $1: 6.0$ & $1: 6.5$ & $1: 4.8$ & .048 \\
\hline Doctor/patient ratio & $1: 1.3$ & $1: 5.1 *$ & $1: 4.9^{*}$ & .001 \\
\hline Respiratory ICU beds/general population ratio & $1: 1,410,000$ & $1: 1,125,000$ & $1: 750,000^{*}$ & .001 \\
\hline \multicolumn{5}{|c|}{$\begin{array}{l} \pm \text { values are mean } \pm \mathrm{SD} \\
* \text { Post hoc Bonferroni test versus the first period, } P<.01 \text {. No significant differences were observed between the second and third periods of time in any variable. } \\
\text { APACHE }=\text { Acute Physiology and Chronic Health Evaluation } \\
\text { (From Reference } 34 \text {, with permission.) }\end{array}$} \\
\hline
\end{tabular}

Table 5. Patient Outcomes in 5 Respiratory ICUs Over a 15 Year Study Interval

\begin{tabular}{|c|c|c|c|c|}
\hline & $1991-1995$ & $1996-2000$ & $2001-2005$ & $P$ \\
\hline Respiratory ICU stay, mean \pm SD d & $23 \pm 4$ & $30 \pm 4 *$ & $32 \pm 3^{*}$ & .004 \\
\hline In hospital mortality $\dagger$ & $82(9)$ & $138(14)$ & $184(15)$ & .049 \\
\hline 3 month mortality & $90(11)$ & $119(14)$ & $152(14)$ & $<.05$ \\
\hline Weaning success & $486(87)$ & $493(74)$ & $524(66)$ & $<.001$ \\
\hline Discharge destination & & & & $<.001$ \\
\hline Home & $178(22)$ & $125(15)$ & $104(10)$ & \\
\hline Nursing home & $21(2)$ & $36(4)$ & $48(5)$ & \\
\hline Acute hospital & $47(6)$ & $95(11)$ & $103(10)$ & \\
\hline Rehabilitation unit & $573(70)$ & $591(70)$ & $780(75)$ & \\
\hline Respiratory status of home discharge patients & & & & $<.001$ \\
\hline Home ventilation via tracheostomy & $22(12)$ & $32(25)$ & $34(33)$ & \\
\hline Home noninvasive ventilation & $8(4)$ & $7(6)$ & $11(11)$ & \\
\hline No ventilatory support & $149(84)$ & $87(69)$ & $59(56)$ & \\
\hline
\end{tabular}

Comparative analysis over the years 1997 to 2006 defined a progressive increase in the utilization of post-acute LTAC services, from $3.3 \%$ of ICU patients requiring MV in 1997 to $8.7 \%$ in 2006. During that same time interval, the percentage of critical care hospitalizations ending in transfer to SNF/IRF remained relatively constant, while the percentage of critical care hospitalizations ending in discharge to home decreased. Acute care hospital mortality rates for the cohort were remarkably constant over this entire time interval. The final discharge destination after transfer changed over time, with more patients discharged to an SNF or rehabilitation facility (19.9\% in 1997-2000, $34.9 \%$ in 2004-2006), and fewer patients discharged to home (32.3\% in 1997-2000, 27.4\% in 2004-2006). Nearly $20 \%$ of the patients were transferred back to an acute care facility, a figure that decreased over time (19.6\% in 1997-2000, $14.2 \%$ in 2004-2006). Although highly informative, this analysis of Medicare beneficiaries is subject to some bias, as the cohort is restricted to individuals $>65$ years old. 
Table 6. Discharge Location of Fee-for-Service Medicare Beneficiaries in Calendar Year 2006

\begin{tabular}{lcccc}
\hline \hline \multicolumn{1}{c}{ Patients* } & $\begin{array}{c}\text { Died in } \\
\text { Hospital } \\
(\%)\end{array}$ & $\begin{array}{c}\text { Discharged } \\
\text { to Home } \\
(\%)\end{array}$ & $\begin{array}{c}\text { Discharged } \\
\text { to SNF/IRF } \\
(\%)\end{array}$ & $\begin{array}{c}\text { Discharged } \\
\text { to LTAC } \\
(\%)\end{array}$ \\
\hline All ICU n & 15.3 & 57.7 & 24.6 & 2.5 \\
Mechanically ventilated & 45.7 & 20.6 & 25.1 & 8.7 \\
* Patients were $>65$ years of age and required $>1$ ICU day and/or mechanical ventilation. \\
SNF = skilled nursing facility \\
IRF = in-patient rehabilitation facility \\
LTAC = long-term acute care hospital \\
(Data from Reference 10.)
\end{tabular}

Lilly et al have summarized a more diverse critical care population of patients discharged from 271 ICUs and 188 non-federal United States hospitals during calendar year $2008 .{ }^{35}$ The mean age of the study population was $62.9 \pm 17$ years. MV was required in $27 \%$ of the population, in contrast to $13 \%$ of the Medicare population. The duration of $\mathrm{MV}$ in the cohort was a mean of 3.8 days. For 176,302 hospital discharges with an ICU admission, the acute care hospital mortality rate was $9.5 \%$, with $58.9 \%$ of discharged patients going home, $9.2 \%$ to an SNF, $3.1 \%$ to a rehabilitation facility, $4.4 \%$ of patients were discharged to other hospitals, and $8.8 \%$ to other types of supportive care. These data show general similarity to the Medicare cohort for patients who require ICU care in general. Clearly, ICU patients in general, short-term MV patients, and especially PMV have a very high demand for PAC services. The vast majority of PMV patients will participate in PAC services during the course of their illness in most published series.

\section{Long-Term Acute Care}

The LTAC category of PAC providers was established by the Centers for Medicare and Medicaid Services (CMS) under the Tax Equity and Fiscal Responsibility Act in 1998, to address inequities in the PPS for the care of chronically critically ill patients. The original concept of the LTAC payment model was to provide a cost based reimbursement for the care of patients who required prolonged acute care following critical illness. In 2003, over concerns related to a rapid expansion in LTAC facilities and related costs, Medicare converted LTACs to an In-patient Prospective Payment System (IPPS) methodology, based on the patient's diagnosis and the facilities wage index, similar to the PPS in STAC hospitals. Payment rates by Medicare for LTACs are based upon the Medicare severity long-term care DRGs (MS-LTC-DRGs), which are identical to the STAC PPS with adjusted relative weights. CMS payment policy treats cases that are discharged from a STAC directly into an LTAC as 2 separate stays, eligible for STAC-PPS payment for the initial hospitalization plus a second, often larger LTAC-PPS payment. LTACs receive a higher payment per case for a similar DRG ( $\sim 7$ times greater), although a lower payment per day. LTAC payments are currently adjusted down for short stay outliers (33\% of discharges in 2006), up for high cost outliers (14\% of discharges), and are also adjusted for interrupted stays. A short stay outlier is a patient with a shorter stay $(<5 / 6)$ than the average stay expected for an MS-LTC-DRG. Under the "interrupted stay" policy, patients discharged from an LTAC to home, STAC, SNF, or IRF, and who are subsequently readmitted to the same LTAC prior to a threshold number of days do not receive an additional LTAC DRG payment. The goal of this policy is to prevent patients from transferring between PAC sites for the purpose of receiving additional reimbursement. The outlier policy for LTACs typically has a lower fixed cost threshold but still only provides for reimbursement of $80 \%$ of subsequent costs.

Since 2003, LTACs have been one of the most highly scrutinized areas of the PAC segment of healthcare because of their substantial growth. Medicare is the predominant payer for LTAC services accounting for the majority of LTAC discharges. ${ }^{36}$ LTACs may be either free-standing or co-located within other hospitals as "hospitals within hospitals" (HwH) or satellites. For profit LTACs account for $81 \%$ of all Medicare discharges from LTAC hospitals. ${ }^{37}$ Low margin LTACs care for a disproportionate share of high cost outliers and patients who have a shorter stay, as both types of patients are "penalized" in the LTACPPS. Despite the extensive LTAC industry regulation, under the initial cost based system of reimbursement, and the current LTAC-PPS based system, the LTAC industry has remained a profitable enterprise. That, combined with a large and willing referral base, has fueled continued growth of the industry.

Currently LTACs under Medicare regulation must meet the following 5 requirements for patient care:

- Provide in-patient services, by or under the supervision of a physician, to Medicare beneficiaries whose medically complex conditions require a long hospital stay and programs of care provided by a long-term care hospital.

- Have an average in-patient stay of $>25$ days for all Medicare patients.

- Have a patient review process, documented in the patient medical record, that screens patients prior to admission for appropriateness of admission to a long-term care hospital, validates within 48 hours of admission that patients meet admission criteria for long-term care hospitals, regularly evaluates patients throughout their stay for continuation of care in a long-term care hospital, 
and assesses the available discharge options when patients no longer meet such continued stay criteria.

- Have active physician involvement with patients during their treatment through an organized medical staff, physician-directed treatment with physician on-site availability on a daily basis to review patient progress, and consulting physicians on call and capable of being at the patient's side within a moderate period of time.

- Provide interdisciplinary team treatment for patients, requiring interdisciplinary teams of healthcare professionals, including physicians, to prepare and carry out an individualized treatment plan for each patient.

LTACs are distinct from other PAC venues in a number of important ways, including a unique case mix because of the high acuity of the patient population. Even within LTACs, however, the case mix can vary based upon the distribution of principal diagnoses. In contrast to the STAC environment, LTACs have a high concentration of acute patients with problems more characteristic of a chronic care environment, including infection control complications, chronic wound care management, and catheter related complications. ${ }^{38}$

LTACs do not provide care exclusively to patients with PMV. The most frequent LTAC diagnosis is MS-LTCDRG 207, which represents a respiratory diagnosis with ventilator support for 96 hours or more (Table 7). ${ }^{39}$ However a broad range of admission diagnoses are recognized, with $11.7 \%$ of patients in 2004 to $15.5 \%$ of patients in 2008 requiring MV. ${ }^{36}$ LTACs, therefore, provide a much broader range of care than required by the PMV patient. About $80 \%$ of LTAC admissions have a preceding acute care hospital stay, and patients who use LTACs have shorter acute care hospital stay than similar patients who do not use LTACs. ${ }^{37}$ To discourage patient shifting between host hospitals and their HwH or satellites, CMS for 2005 proposed a "25\% rule." This rule would establish payment adjustments (reductions) to limit the percentage of patients who could be readily transferred from a host hospital to their LTAC facility. In July of 2007, CMS extended the $25 \%$ rule to all LTACs. Subsequent legislation (Medicare, Medicaid and State Children's Health Insurance Program [SCHIP] Extension Act of 2007) suspended implementation of the $25 \%$ rule for both free-standing and $\mathrm{HwH}$ facilities. The 2007 legislation limited the proportion of Medicare patients who can be admitted from a $\mathrm{HwH}$ or satellite host to $50 \%$ for 3 years. This legislation also began a 3 year moratorium on the creation of new LTAC facilities and beds, with certain exemptions.

Two recognized factors have heightened scrutiny of LTACs, starting in 2003. First, as previously noted, the number of LTACs tripled from 105 facilities in 1993 to 318 in 2003. Not surprisingly, Medicare spending for
Table 7. Top 10 Principal LTAC Diagnoses for Fiscal Year 2010

\begin{tabular}{|c|c|c|c|}
\hline DRG & Diagnosis & $\begin{array}{c}\text { Total } \\
\text { Discharges } \\
\text { per DRG }\end{array}$ & $\begin{array}{l}\text { National } \\
\text { Average } \\
\text { of Stay } \\
\text { for DRG } \\
\text { (d) }\end{array}$ \\
\hline 207 & $\begin{array}{l}\text { Respiratory system diagnosis with } \\
\text { ventilator support } \geq 96 \mathrm{~h}\end{array}$ & 16,236 & 36.8 \\
\hline 189 & $\begin{array}{l}\text { Pulmonary edema and respiratory } \\
\text { failure }\end{array}$ & 11,349 & 22.9 \\
\hline 871 & $\begin{array}{l}\text { Septicemia without mechanical } \\
\text { ventilation for } \geq 96 \mathrm{~h} \text { with major } \\
\text { complications and comorbidities }\end{array}$ & 7483 & 22.7 \\
\hline 177 & $\begin{array}{l}\text { Respiratory infections and } \\
\text { inflammations with major } \\
\text { complications and comorbidities }\end{array}$ & 5,095 & 23.2 \\
\hline 592 & $\begin{array}{l}\text { Skin ulcers with major } \\
\text { complications and comorbidities }\end{array}$ & 3,604 & 27.1 \\
\hline 949 & $\begin{array}{l}\text { Aftercare with complications and } \\
\text { comorbidities/major complications } \\
\text { and comorbidities }\end{array}$ & 3,111 & 22.2 \\
\hline 208 & $\begin{array}{l}\text { Respiratory system diagnosis with } \\
\text { ventilator support }<96 \mathrm{~h}\end{array}$ & 2,929 & 17.5 \\
\hline 193 & Simple pneumonia and pleurisy & 2,878 & 20.4 \\
\hline 190 & $\begin{array}{l}\text { COPD with major complications and } \\
\text { comorbidities }\end{array}$ & 2,685 & 20.6 \\
\hline 539 & $\begin{array}{l}\text { Osteomyelitis with major } \\
\text { complications and comorbidities }\end{array}$ & 2,453 & 31.0 \\
\hline DRG & $\begin{array}{l}\text { nosis-related group } \\
\text { nce } 39 \text {, with permission.) }\end{array}$ & & \\
\hline
\end{tabular}

LTACs also grew, at a rate of $15 \%$ per year, with total payments rising from $\$ 398$ million in 1993 to $\$ 1.9$ billion in $2001 .{ }^{40}$ Second, LTACs are not evenly distributed across the United States (Fig. 4). LTAC hospitals are concentrated in the Northeast and South, providing very high LTAC bed to beneficiary ratios in certain regions of the United States and relatively low ratios in other regions. ${ }^{40}$ In the absence of data to support an uneven distribution of CCI patients, this discrepancy suggests that alternative models of care for CCI patients currently exist based upon geographic distribution rather than clinical need. Logically, a reviewer must assume that medical conditions treated in LTAC hospitals are treated in acute care hospitals where no LTACs operate. This raises the question as to which model of PAC is optimal, from both a clinical and cost perspective.

For the severely ill patient, LTACs may provide an alternative to continued care in the ICU or specialized care unit in the acute care hospital, and offer the acute care hospital motivation to control hospital days and capture additional payment revenue from 2 DRG payments. For the less severely ill patient, LTACs could be an alternative to the use of other less expensive PAC services, including SNFs and IRFs. Both the payer (Medicare) and the pro- 


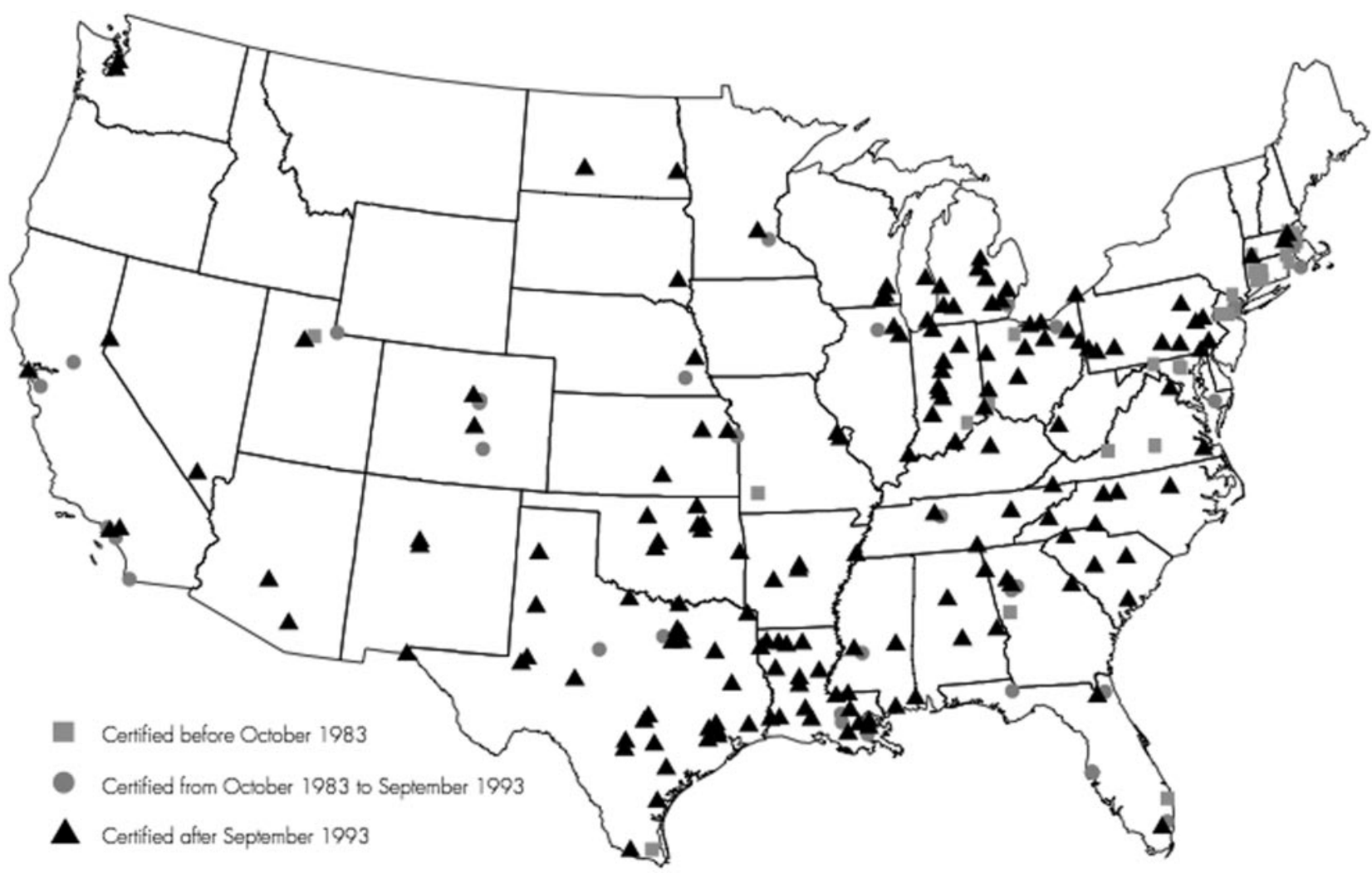

Fig. 4. Distribution of long-term acute care facilities across the United States in calendar year 2004. (From Reference 40.)

vider (LTACs) have struggled over the past $\sim 8$ years to provide convincing evidence of a cost or clinical benefit to this care model.

Two provider-based studies have been subject to peer review and addressed the role of the LTAC model of care for critically ill patients. The Ventilation Outcomes Study Group (sometimes called the Barlow studies), sponsored by the National Association of Long-Term Hospitals (NALTH), provided patient characteristics and clinical outcomes of 1,419 patients referred to 23 LTAC hospitals for weaning from March 1, 2002, to February 28, 2003.13,41 Eleven hospitals were free-standing and 12 were $\mathrm{HwH}$. The median number of beds per hospital was 50, with a range of 15 to 311 . Five of the LTACs had ICU bed capacity. Registered nurse staffing ranged from 3 to 10 patients per nurse, and respiratory therapist staffing ranged from 2 to 20 patients per therapist. The prolonged ventilator patient population ranged from $1 \%$ to $50 \%$ of total admissions, with an average value of $10 \%$. Weaning protocols were employed in $<50 \%$ of the facilities. This "snapshot" of LTAC facilities suggested a wide variability in patient care services within the LTAC model of care. PMV patients in this cohort had a high frequency of tracheotomy tubes $(94.7 \%)$, indwelling Foley catheters (94.5\%), enteral feeding tubes $(92.5 \%)$, and pressure ulcerations $>$ stage II $(42 \%)$. The mean APACHE III acute physiology score was 35 (range 4-115), and only $70 \%$ of patients on admission were able to follow commands. The median transferring hospital stay was 27 days (range $0-563 \mathrm{~d}$ ), and the median days on MV at the referral institution was 27 days (range $0-1,154 \mathrm{~d}$ ). Renal replacement therapy was required in $5.6 \%$ of the admissions. Based upon the clinical description of the patient population in this cohort, the PMV patients in an LTAC facility are complex patients with clear acute care needs at high risk for subsequent complications.

The most common complications of the LTAC admissions in this cohort were infections. The time to wean from MV was significantly prolonged in patients who developed infectious complications in the LTAC. The need for patient isolation for particularly resistant bacterial isolates was frequent: methicillin-resistant Staphylococcus aureus in 453 patients $(31.9 \%)$, vancomycin-resistant enterococcus in 85 patients $(6.0 \%)$, and 55 patients $(3.0 \%)$ with both organisms. Isolation for other infections was noted in 827 patients $(58.2 \%)$.

Weaning success in this cohort ranged from $42 \%$ to $83 \%$ by facility, and the LTAC hospital mortality ranged from $0 \%$ to $47 \%$ by facility ( $25 \%$ overall). One-year mortality was $63 \%$, which is within the range of other singlecenter studies of LTAC outcomes. 
Nearly $20 \%$ of patients were discharged to a short-stay acute hospital for surgery, a procedure, or treatment of an intercurrent illness; $65 \%$ of those were ventilator dependent at the time of discharge. The majority, 144 of 207 patients $(69.6 \%)$, were transferred for intercurrent medical problems unable to be treated at the LTAC. Forty-seven of the 207 patients $(22.7 \%)$ were transferred to STAC hospital for surgery or a procedure. Six patients were transferred at the request of the patient and/or family; 10 patients were transferred for unknown reasons. The transfer of patients to SSAHs for treatments not provided at the LTAC may affect outcomes in 2 ways: increase in ventilator-dependent discharges credited to the LTAC, and decrease in mortality as an outcome at the LTAC.

Fourteen LTACs from the cohort submitted cost data, for 978 patients. The mean cost of care for patients at all LTACs reporting financial data were $\$ 63,672$ (median $\$ 47,217$, range $\$ 949$ to $\$ 553,485$ ). A similar mean cost of $\$ 56,825$ (in 1994 dollars) in PMV patients discharged from 26 LTACs was reported from a large for-profit healthcare system. ${ }^{14}$ The investigators attempted to compare the cost of LTACs to acute care hospitals by extrapolating from the Dasta et al data. ${ }^{12}$ Using a figure of $\$ 3,968$ per hospital day ( $>$ day 3) and incremental ventilator day costs of $\$ 1500$ per day, the projected acute care costs for the same stay in the LTAC cohort would be $\$ 210,304$. The validity of this comparison is highly questionable, based upon the assumption of a constant stay in both environments and the inclusion of the highest cost ICU days in the comparison data. A more appropriate comparison of care venues might be the costs of LTAC care and acute subspecialty or step-down care continued in the STAC hospital.

The Ventilation Outcomes Study Group provided a valuable contribution to the debate regarding LTAC care by providing substantial clinical detail on the severity of illness and comorbidities of LTAC PMV patients. The PMV patient population is a smaller, yet variably sized component, of the overall LTAC care model. PMV referrals to LTAC hospitals are characterized by a high level of comorbidities, numerous infection control complications, high device utilization, and long STAC hospital lengths of stay prior to transfer. These data highlighted the difficulty of finding an appropriate comparison group between PMV patients admitted to an LTAC and PMV patients not admitted to an LTAC for comparative cost analyses.

Votto and colleagues described their experience with an LTAC demonstration project at St Francis Medical Center in Hartford, Connecticut. ${ }^{15}$ The LTAC group consisted of all patients admitted directly from an acute care hospital to the study LTAC and discharged from the LTAC during a 24 month period from September 2004 to August 2006 (Table 8). The control population was taken from a review of all hospital discharges from St Francis Hospital and Medical Center during fiscal year 2002, with a $>30$ day
Table 8. Comparison Between LTAC Patients From the Hospital for Special Care (Connecticut) and a Retrospective Cohort Not Managed in the LTAC

\begin{tabular}{lcc}
\hline \hline & $\begin{array}{c}\text { Control } \\
(n=187)\end{array}$ & $\begin{array}{c}\text { LTAC } \\
(n=196)\end{array}$ \\
\hline $\begin{array}{l}\text { Died, no. (\%) } \\
\text { Discharged home, no. (\%) }\end{array}$ & $20(17.6)$ & $26(13.3)$ \\
Discharge to SNF, no. (\%) & $73(39.0)$ & $61(31.1)^{*}$ \\
Mean daily cost per patient, & $\$ 1,520 \pm \$ 559$ & $40(20.4)^{*}$ \\
$\quad$ mean \pm SD & $\$ 1,154 \pm \$ 376^{*}$ \\
Mean total cost per patient & $\$ 59,103$ & $\$ 36,626$ \\
$\begin{array}{l}* P<.05 . \\
\text { LTAC }=\text { long-term acute care hospital } \\
\text { SNF }=\text { skilled nursing facility } \\
\text { (Data from Reference 15.) }\end{array}$ & & \\
& & \\
\hline
\end{tabular}

stay and comparable DRGs. From this group, 187 patients who met standard LTAC admission guidelines were selected to create a control group. The LTAC population had a statistically insignificant reduction in mortality rate, a significantly greater rate of home discharge (31\% vs $11 \%$ ), a reduced rate of SNF discharge (20\% vs $39 \%$ ), and a significantly reduced cost per hospital day $(\$ 1,154$ vs $\$ 1,520)$. The mean costs for hospitalization in the LTAC were significantly reduced, compared to the control population $(\$ 36,626$ vs $\$ 59,203)$.

Cost analyses per hospitalization in the cohort were very similar to those from the Ventilation Outcomes Study Group. ${ }^{41}$ Although favorable to the LTAC model of care from a cost perspective, both of these analyses suffer from the retrospective nature of the control group and limited data to assure carefully matched populations. Cost comparisons appear to include the entire spectrum of acute care in the control population, rather than a comparable clinical interval selected from the acute care hospitalization to match the long-term care hospitalization. Finally, data analyses isolated to the LTAC only, fail to address any cost implication associated with patient discharge disposition. Patient return to STAC hospitals or discharge to SNF introduce incremental costs to the healthcare system, so patient outcome becomes a critical contributor to the success of a PAC delivery model when the issue of cost is considered.

Payers, in this case CMS, are also interested in confirming a benefit to the LTAC care model. In the absence of adequate peer reviewed data, Medicare has relied on internal and consultant review for policy determination. The Medicare Payment Advisory Commission (MedPAC) is an independent congressional agency established by the Balanced Budget Act of 1997 to advise the United States Congress on issues affecting the Medicare program. A MedPAC report, initially presented in 2003, documented rapid growth in the number of LTACs and total Medicare 
spending on LTAC services, high concentrations of LTACs in a few states, with higher payments and lack of evidence for better clinical outcomes for LTAC users.

This initial MedPAC report prompted a study from the Lewin Group in an analysis for the NALTH, using claims data from 1998-2000, which illustrated the complexity of matching patient data between LTAC treated and nonLTAC treated patients. ${ }^{37}$ The Lewin report examined 5 outcome parameters over a 180 day episode of care, using 3 different comparative models between patients receiving LTAC care and non-LTAC care. The analysis considered the entire spectrum of LTAC care, without consideration for severity of illness. Very different conclusions were reached based upon the model used to match the patient populations between LTAC and non-LTAC care. This report is primarily of interest because it illustrates the challenge in matching populations of LTAC and non-LTAC treated patients for a comparative analysis of outcome and/or costs in the absence of randomization. The actual cost calculations for this report predated the implementation of the LTAC PPS, so they are not relevant to the current care model, but the variable interpretation based upon modeling techniques provides substantial insight into the current controversy over studies that attempt to define the benefits of LTAC care to the PMV patient population.

A subsequent report from MedPAC (2004), using multivariate modeling on a full sample of LTAC claims, suggested that different outcomes with LTAC care occur based upon the severity of illness in the patient population. ${ }^{40}$ MedPAC reported on a subset of cases referred for LTAC care: those scoring above the 95th percentile in a model of the probability of LTAC use, and those with a tracheostomy and long-term ventilator support. Episode payments were found to be lower for episodes using LTAC in this population than for others, and the difference was statistically significant. Among patients within the top 5\% probability of using LTAC care, LTAC users and non-users had statistically similar episode payments. The report found fewer acute readmissions among those discharged into LTACs, compared to those discharged into other post-acute settings. Further, it found a reduction in acute hospital stay associated with LTAC use, having 7 fewer days in the full sample and 9 fewer days in the most clinically complex group. These results apply only for the most complex LTAC admissions, not for all LTAC admissions. The difference between the 2003 and 2004 conclusions emphasizes the importance of identifying clinically appropriate comparison groups for the CCI patients seen in LTACs.

CMS has subsequently contracted RTI International for a continued assessment of PAC in the ICU population. ${ }^{36,37}$
The most recent report has been published for review in June 2011.36 The analysis continues to use a pseudorandomization technique that employs a propensity scoring model to match patient populations with an equal likelihood of being referred for LTAC care, based upon categorized clinical characteristics. From a total of 74 selected MS-DRGs with a high rate of LTAC referral, patients were categorized into 7 conditions: ventilator; infections; aftercare, wound, and skin care; complex rehabilitation; pneumonia; heart failure; and COPD/other respiratory failure. The report constructed episodes of care using all 2007 Medicare Provider Analysis and Review (MedPAR) in-patient claims, searching within 60 days of admission and discharge dates to a general acute care hospital to find claims from other admissions-including those to LTACs, SNFs, and IRFs for the same Medicare beneficiary to create an analysis of the episode of care. For statistical analysis, they matched non-LTAC users to each LTAC user with predicted probabilities that were closest in value to the propensity scores of the cases. The matching with propensity scores assumes that LTAC and nonLTAC users will have similar clinical characteristics, including similar acuity. By using non-LTAC controls from hospitals with low LTAC referral patterns (many of which are hospitals located in parts of the country with few LTACs), they expected to minimize the possibility that non-LTAC controls were similar to LTAC cases in observed characteristics but different from them in some important but unobserved characteristics.

Based upon this modeling, they identified that patients transferred to LTACs had longer stays, higher total payments, and higher provider costs than clinically similar patients who completed their acute care during the index general acute care hospital admission. This finding was present for all 7 condition groups. Both payments and costs were estimated to be significantly higher for LTAC users. The magnitude of the differences varied; significant positive differences were found, however, for every patient subgroup.

Patients in the ventilator condition group showed the smallest proportional differences in Medicare stays, payments, and costs between LTAC users and non-users of the study groups (Fig. 5). The episode-level payments and costs for patients referred to LTAC were significantly higher than those who were not referred to LTACs (top panels). The episode payment differences are expected, as the LTAC referral generates an additional Medicare payment, in comparison to patients who remain in the index hospital. In the percent differences in episode payment-to-cost ratio panel (bottom left), the episode payment-to-cost ratio is significantly higher for the LTAC episodes than for the acuteonly episodes, with a low likely LTAC referral, but for the more likely LTAC referral, the episode payment-to-cost ratio is not significantly different between the 2 types 

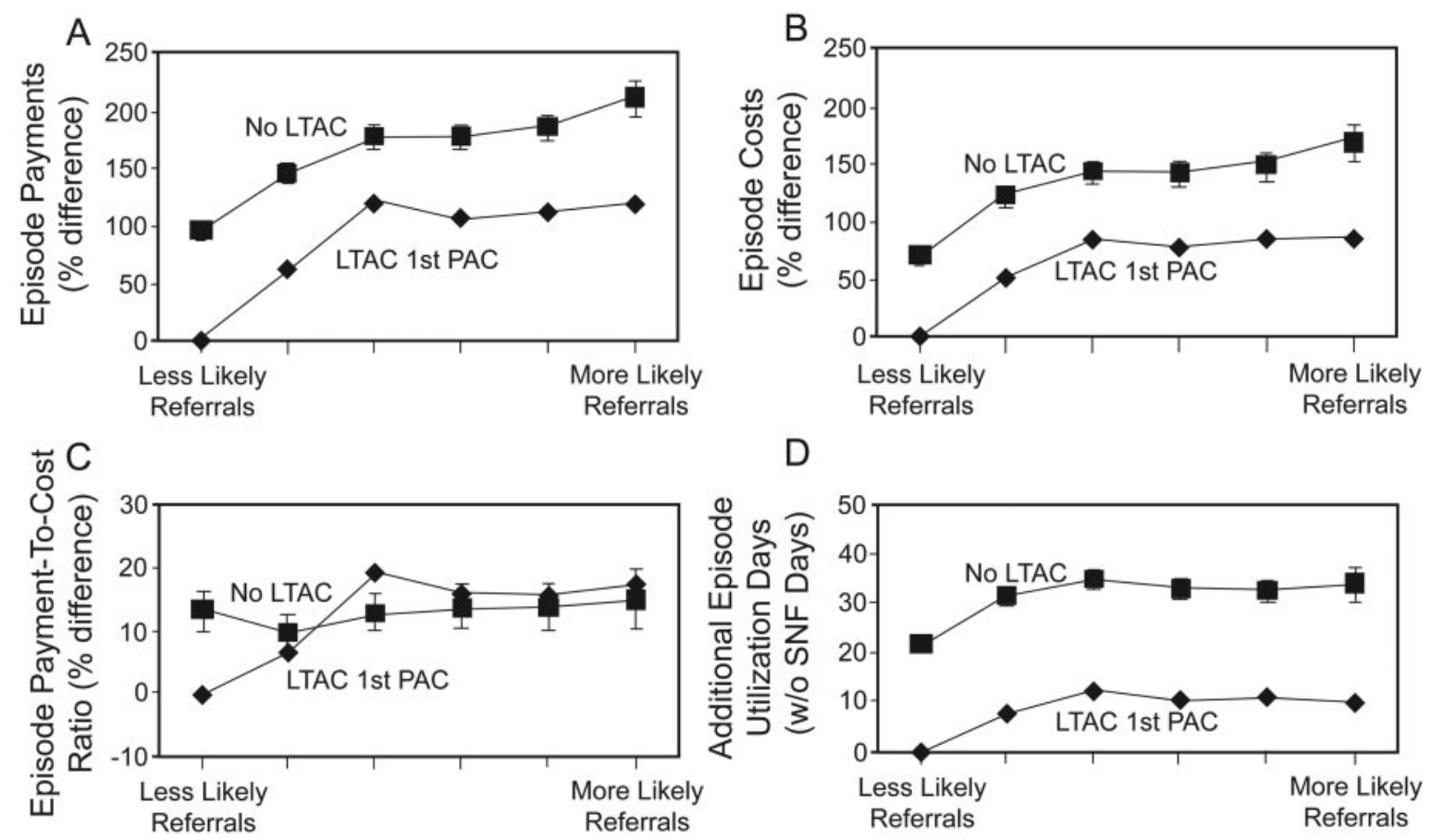

Fig. 5. Comparative outcomes for ventilator group patients between episodes with and without long-term acute care (LTAC) hospital referral. The panel graphs the effect of LTAC referral on ventilator group parameters, where the effect (the percent difference) is relative to a "reference case" that is a non-LTAC user in the lowest category of LTAC referral. A: Mean payment of $\$ 29,494$ for reference group. B: Mean payment of $\$ 32,930$ for reference group. C: Mean ratio of 0.90 for reference group. D: Mean of 21.6 days for reference group. Panel $D$ includes actual days rather than percentage differences. PAC $=$ post acute care. SNF $=$ skilled nursing facility. (From Reference 36 , with permission.)

of episodes. The higher costs for the LTAC-referral episodes are offset by their higher payments; and for those patients most likely to be referred to LTACs, the investigators noted no difference in the overall profitability of episodes of care between LTAC and non-LTAC patients. In the additional episode utilization days (without SNF days) panel (bottom right), the difference in total episode days (not including SNF days) are plotted. LTAC users have at least 20 more days of in-patient care than clinically similar patients who do not use LTACs, and this difference was seen across all levels of likelihood of LTAC referral.

The investigators attempted to determine if SNF days substituted for LTAC days. The report suggested that $41.2 \%$ of LTAC cases and $42.7 \%$ of matched non-LTAC controls had an SNF stay within the episode. SNF stays were the same or longer for LTAC users compared to non-users in 5 out of the 7 condition groups. In the ventilator care group the non-LTAC users averaged only about 1.5 more days in SNFs than did LTAC users.

The report acknowledges that unmeasured selection factors could affect the likelihood of LTAC use, from both the index acute care hospital and the LTAC perspective. Acute care hospitals may use referral criteria or physician judgment that cannot be captured from coded data sets. Alternatively, LTACs are seeking patients who fit their clinical expertise, but must also fit within regulatory restrictions that favor long-staying patients $(>25 \mathrm{~d})$ and penalize shorter-than-expected stays. These transfer decisions may influence LTAC utilization in ways that are not easily predicted from the reported data and create a casemix difference that cannot be appreciated.

The consensus of independent MedPAC and RTI International reports is that CMS should seek to define the critically ill, medically complex patient as a necessary first step in determining how Medicare should appropriately pay for such patients, regardless of whether those patients are treated in a general acute care hospital, an LTAC, or any other setting. CMS is expanding its data collection to better characterize the CCI and PMV population, comparing models of care delivery for outcome and cost implications, with a goal to develop payment models that would reimburse care for these patients' reasonably and appropriately in LTACs or any other site of care. Conclusions suggested by these reports include:

- An LTAC admission is associated with a shorter stay in the acute care hospital after controlling for age, sex, critical care use, and comorbid conditions. 
- LTACs provide a service that is comparable to general acute step-down units in many regions of the country and that is not unique to LTACs.

- Medicare patients with respiratory conditions requiring MV make up only one fraction of all LTAC patients $(\sim 15-20 \%)$.

- A substantial percent of LTAC admissions qualify for a payment reduction as a "short stay outlier," suggesting a possible misclassification as a long stay patient, although this designation is complicated by "short stay outliers," including patients who die in the LTAC and those who require readmissions to acute care.

Unresolved in the conclusions from the consultant reports is how the reported differences in cost and outcome reflect differences in the care delivery model (LTAC) as compared to unmeasured case-mix differences.

Despite their intensive scrutiny, LTAC hospitals have assumed a substantial burden of care for STAC hospitals especially for patients with PMV. Detailed cost comparisons between STAC hospitals and LTAC hospitals will always be limited by the accuracy of creating a comparative case-mix in the absence of randomization. The appropriate outcome variables to consider in the analysis are also important. A simple comparison of mortality is a complex outcome parameter to consider in the PMV population. Given the poor long-term survival and quality of life noted in the PMV population, mortality may reflect a good outcome if consistent with the patient's care beliefs. Given the expected expansion in the CCI population, and PMV patients in particular, attention should focus to improving the efficiency of the STAC and LTAC care model for the CCI population. As of now, the LTAC industry operates under a complex range of restrictions that are not patient focused. LTAC hospitals, based upon the limited data reported in the public domain, demonstrate variability in their care delivery models, which may be related to case-mix variability or other undefined variables. The LTAC industry has the potential to make important contributions to defining efficient care models for the CCI patient population, based upon their access to a large segment of the PMV population.

\section{Skilled Nursing Facilities}

In comparison to the acute and long-term acute care environments, relatively limited data are available on the outcome and cost of CCI patients in SNFs. A few studies have focused on the patient with PMV, but very limited cost information is provided. Lindsay and colleagues reported their outcomes with PMV patients referred to a stand-alone nursing home ventilator unit. ${ }^{42}$ Sixty-eight
(67\%) of 102 patients were successfully weaned during a 6-year period. NIV facilitated successful weaning in $27(26 \%)$ of 102 patients. Of the 28 chronic ventilatordependent patients admitted with a neuromuscular etiology for respiratory failure, NIV was utilized in $73 \%(8 / 11)$ of the successfully weaned patients. Total variable costs per ventilator per patient per day for the years 1998-2000 were reported as $\$ 319.79, \$ 302.75$, and $\$ 297.59$. Six-year cost savings for referring hospitals were estimated at $\$ 18.5$ million.

Despite the limitation in available data, published Medicaid payment rates to skilled facilities are significantly reduced, compared to alternative care models, even for patients with the highest acuity. ${ }^{43}$ For SNFs the reduction in patient costs are not the primary issue of concern for the PMV population. The primary concern is that payment levels from Medicare ( $\sim 20 \%$ patients) and Medicaid ( $\sim 80 \%$ patients) might not provide the necessary resources to provide optimal patient care. Medicare patients with PMV are classified under extensive service resource utilization groups that provide higher reimbursement per day but that is markedly low, compared to anticipated costs in complex critically ill patients. Medicaid reimbursement rates are typically in the range of $\$ 100-120$ per day. ${ }^{43}$ Reimbursement rates inadequate to support patient care in the environment, particularly for patients with elevated acuity, could lead to unacceptable rates of readmission to the STAC environment and incremental costs for the episode of care. ${ }^{44}$

\section{Episodes of Care}

Many of the previous studies have viewed patients with PMV from the perspective of individual care units. STAC outcomes are considered independent of the availability and utilization of PAC facilities. Reported LTAC outcomes, both for mortality and cost, isolate the LTAC hospitalization. These reports fail to consider the important interrelationships between these facilities over the care spectrum for a given PMV episode of care. These individual care unit reports provide only some insight into the relationships between acute and post acute care venues for the PMC population.

Transitions in care are driven by both clinical and payer variables. LTAC facilities accept $\sim 90 \%$ of their patient population from STAC facilities, often after a very prolonged period $\sim 25-30$ days of STAC hospitalization. Readmissions from LTAC to STAC hospitals occur in the range of $15-20 \%$, but these rates vary widely in published series. A high percentage of PMV patients from both STAC and LTAC hospitals are discharged to SNF facilities, where 


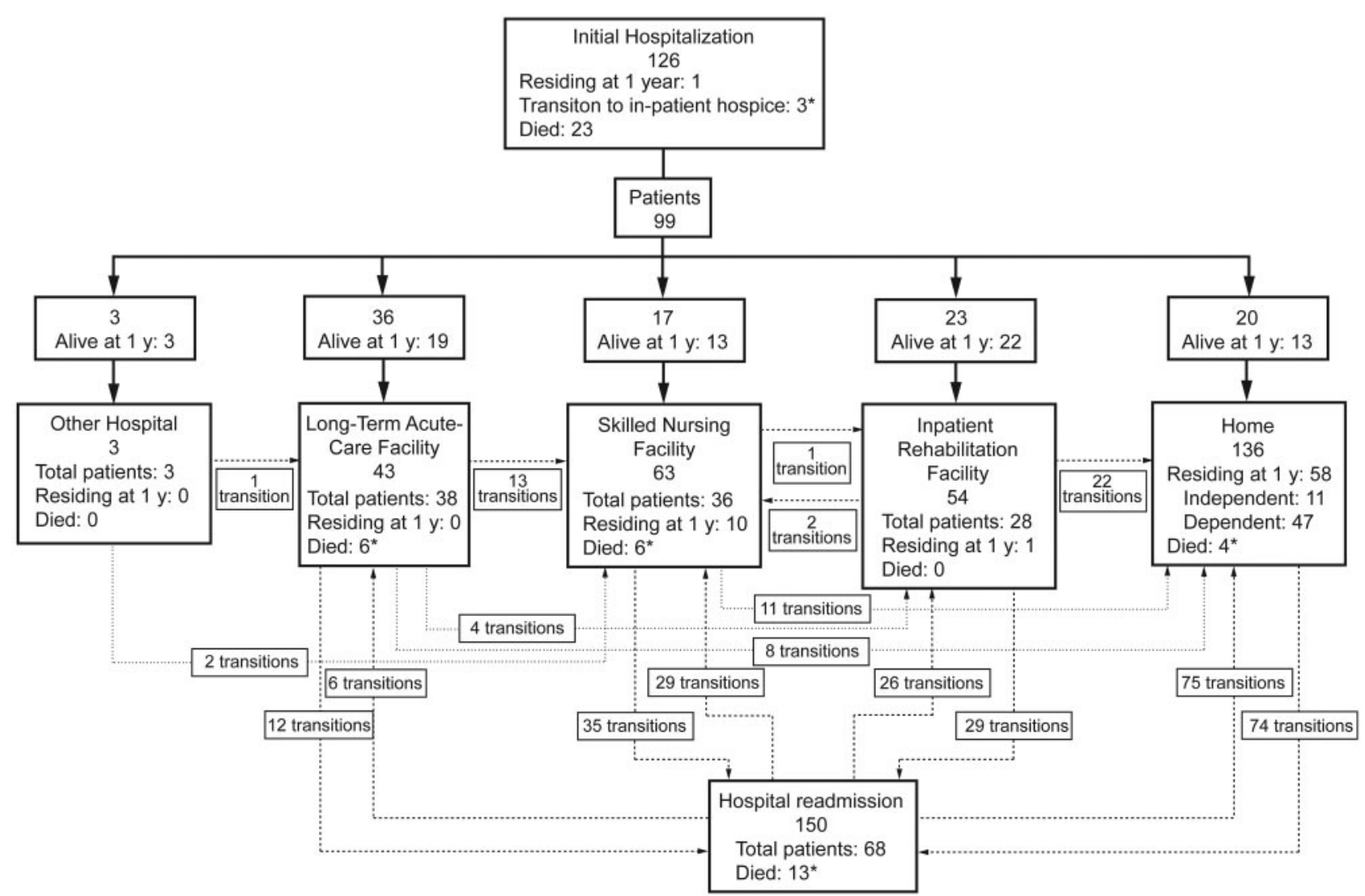

Fig. 6. Trajectories of care for prolonged mechanical ventilation cohort members over the first year post-discharge. The figure depicts 126 patients entering the hospital, with 99 (79\%) discharges (23 died and 1 patient remained in the hospital) then experiencing 457 transitions in care location during follow-up. Arrows between care locations depict both the direction of patient transitions as well as the total number of patients transferred over 1 year between locations. Solid lines represent initial transitions between the hospital and other locations. Dashed lines represent subsequent hospital readmissions and discharges involving post-discharge care locations. Dotted lines represent transitions among post-discharge care locations, including home. Within each box representing a location of care, a summary is provided of the total numbers of both readmissions and patients admitted, as well as how many remained or had died in each at 1 year. ${ }^{*}$ Seven transitions to in-patient hospice (and death) not shown graphically ( 3 from the acute hospitalization and one each from home, long-term acute care facility, skilled nursing facility, hospital readmission). One skilled nursing facility to skilled nursing facility transition not shown. (From Reference 45, with permission.)

the readmission rate to STAC hospitals may also be significant. To understand these interrelationships, greater demand has been placed on considering the PMV patient over the entire care episode.

To address this issue, Unroe and colleagues conducted a 1-year, prospective cohort study in the adult ICUs at Duke University hospital. The investigators enrolled 126 patients, beginning in April 2006, through daily screening of ICUs by study staff, with follow-up completed in April $2008 .{ }^{45}$

Patients were eligible for the study if they were $\geq 18$ years of age and either had received MV for $\geq 21$ days with $<48$ hours of unassisted breathing or $\geq 4$ days of MV with placement of a tracheostomy. Costs for the initial hospitalization were derived from hospital charges and department specific cost/charge ratios. Costs for subse- quent care were estimated by day, using reported venue costs averaged for the referral region.

Mean total 1-year costs of healthcare for cohort members were $\$ 306,135$ (SD \$285,467), while costs for the entire cohort exceeded $\$ 38.5$ million. The majority of these costs ( $\$ 28.1$ million, or $73 \%$ ) were incurred during the STAC hospitalization. The highest mean cost for PAC was for individuals receiving LTAC care $(\$ 91,277)$, followed by care in a SNF $(\$ 31,892)$, in an $\operatorname{IRF}(\$ 21,244)$, and home health service care $(\$ 6,669)$. No differences in 1-year costs by health outcomes grouping were recognized. These patients experienced a median of 4 transitions of care location and spent nearly $75 \%$ of their days alive in hospitals or PAC hospitals, or receiving home care. The transitions in patient care for this cohort are summarized in Figure 6. The study investigators estimated Medicare costs for the 
PMV population 15 times greater than the average Medicare patient, with a $50 \%$ higher readmission rate to acute care facilities.

This study is a landmark contribution to our understanding of the PMV population and their burden to the healthcare system. The 1-year costs reported are more reflective of their true care costs and provide valuable insight into the important interaction of care venues.

\section{Cost Effectiveness}

A cost-effectiveness analysis produces a ratio, such as the cost per year of life gained, where the denominator reflects the gain in health from a specific intervention (eg, life-years gained, number of additional survivors) and the numerator reflects the cost in dollars of obtaining that gain. ${ }^{46}$ Cost-effectiveness analyses describe a case based model with specific modeling parameters and bestavailable estimates of cost and patient outcome, based upon the published literature.

Cox and colleagues modeled the cost-effectiveness of providing MV for at least 21 days to a 65 -year-old critically ill patient, compared to the provision of comfort care resulting in withdrawal of ventilation. ${ }^{47}$ Providing PMV to the patient cost $\$ 55,460$ per life-year gained and $\$ 82,411$ per quality-adjusted life year (QALY) gained, compared to withdrawal of ventilation. Cost-effectiveness ratios were most sensitive to variation in age, hospital costs, and probability of readmission, and less sensitive to PAC facility costs. The incremental costs per QALY gained by PMV provision exceeded $\$ 100,000$ with age $>68$ and when predicted 1-year mortality was $>50 \%$.

\section{Summary}

Current clinical studies of the CCI population are heavily weighted toward the PMV population. The care of the PMV patient occurs over a broad range of acute, long-term acute, skilled, and rehabilitative venues. Important outcomes to patients and their surrogates with respect to decision making, such as long-term independence and quality of life indicators, are being explored for this population, but more work will be needed.

Almost all reported studies of PMV patients are observational in character, with a recognized dearth of intervention trials. The need for intervention trials with detailed cost analysis will require cooperative networks, given the recognized variability in the subject population and relatively small sample size from any single center. The LTAC community is organized to be a leader in this field, but operates under substantial regulatory restriction that complicates management and outcome assessment. Specific cost analysis for intervention trials in the areas of weaning, mobility, wound care, and surrogate decision making would be beneficial to care programs for this population. Optimally, these trials could be conducted over the continuum of care with acute and post acute care partners. These interventions should focus on specific clinical outcome parameters to guide educated decisions by payers and clinicians. The transitions in care venues are frequent, and the factors, independent of current payer models, that both cause and determine these transitions are unknown. It is critical to determine if these transitions are patient or system based. Clinicians who care for these patients frequently compartmentalize their care to the current environment with limited insight into the long-term transitions these patients face in their recovery.

Further observational studies, with more rigorous statistical control for matching of case mix, cost, and outcome assessment, will be required. An important question is whether we should collect "better" studies in the current care model, or develop collaborative care groups to collect critical data and design "best" care models free from the payer restrictions. Support for centers of excellence to study the care management of this population should be enthusiastically considered by payers, given the tremendous financial burden of the population.

For the LTAC care venue, there is a substantial lack of cost and outcome information for the non-PMV population subjected to peer review. This is important when one considers that PMV patients represent the smaller fraction of the LTAC patient population. Discussion of the LTAC model of care should isolate the patients with PMV from other diagnostic groups served by this PAC venue to establish appropriate comparisons. Independent of the current controversy regarding payment issues, a substantial burden of PMV patients is anticipated. The LTAC industry is a valuable contributor to the PAC process, but should assume a burden for investigating its care models to improve quality and reduce costs.

\section{REFERENCES}

1. Meade M, Cook D, Guyatt G, Slutsky A, Arabi Y, Cooper D, et al. Ventilation strategy using low tidal volumes, recruitment maneuvers, and high positive end-expiratory pressure for acute lung injury and acute respiratory distress syndrome: a randomized controlled trial. JAMA 2008;299(6):637.

2. The Acute Respiratory Distress Syndrome Network. Ventilation with lower tidal volumes as compared with traditional tidal volumes for acute lung injury and the acute respiratory distress syndrome. The Acute Respiratory Distress Syndrome Network. N Engl J Med 2000; 342(18):1301-1308.

3. Papazian L, Forel J-M, Gacouin A, Penot-Ragon C, Perrin G, Loundou A, et al. Neuromuscular blockers in early acute respiratory distress syndrome. N Engl J Med 2010;363(12):1107-1116.

4. Peek G, Mugford M, Tiruvoipati R, Wilson A, Allen E, Thalanany $\mathrm{M}$, et al. Efficacy and economic assessment of conventional ventilatory support versus extracorporeal membrane oxygenation for severe adult respiratory failure (CESAR): a multicentre randomised controlled trial. Lancet 2009;374(9698):1351-1363. 
5. The National Heart, Lung, and Blood Institute Acute Respiratory Distress Syndrome (ARDS) Clinical Trials Network. Comparison of two fluid-management strategies in acute lung injury. N Engl J Med 2006;354(24):2564-2575.

6. Erickson SE, Martin GS, Davis JL, Matthay MA, Eisner MD, NIH NHLBI ARDS Network. Recent trends in acute lung injury mortality: 1996-2005. Crit Care Med 2009;37(5):1574-1579.

7. Rivers E, Nguyen B, Havstad S, Ressler J, Muzzin A, Knoblich B, et al. Early goal-directed therapy in the treatment of severe sepsis and septic shock. N Engl J Med 2001;345(19):1368-1377.

8. Nelson JE, Cox CE, Hope AA, Carson SS. Chronic critical illness. Am J Respir Crit Care Med 2010;182(4):446-454.

9. Carson SS, Bach P. The epidemiology and costs of chronic critical illness. Crit Care Clin 2002;18(3):461-476.

10. Kahn JM, Benson NM, Appleby D, Carson SS, Iwashyna TJ. Longterm acute care hospital utilization after critical illness. JAMA. 2010 Jun. 9;303(22):2253-2259.

11. Cox CE, Carson SS, Lindquist JH, Olsen MK, Govert JA, Chelluri L. Differences in one-year health outcomes and resource utilization by definition of prolonged mechanical ventilation: a prospective cohort study. Critical Care 2007;11(1):R9.

12. Dasta JF, McLaughlin TP, Mody SH, Piech CT. Daily cost of an intensive care unit day: the contribution of mechanical ventilation. Crit Care Med 2005;33(6):1266-1271.

13. Scheinhorn DJ, Hassenpflug MS, Votto JJ, Chao DC, Epstein SK, Doig GS, et al. Ventilator-dependent survivors of catastrophic illness transferred to 23 long-term care hospitals for weaning from prolonged mechanical ventilation. Chest 2007;131(1):76-84.

14. Seneff MG, Wagner D, Thompson D, Honeycutt C, Silver MR. The impact of long-term acute-care facilities on the outcome and cost of care for patients undergoing prolonged mechanical ventilation. Crit Care Med 2000;28(2):342-350.

15. Votto JJ, Scalise PJ, Barton RW, Vogel CA. An analysis of clinical outcomes and costs of a long term acute care hospital. J Med Econ 2011;14(2):141-146.

16. MacIntyre NR, Epstein SK, Carson S, Scheinhorn D, Christopher K, Muldoon S, et al. Management of patients requiring prolonged mechanical ventilation: report of a NAMDRC consensus conference. Chest 2005;128(6):3937-3954.

17. Cook CH, Martin LC, Howard B, Flancbaum LJ. Survival of critically ill surgical patients discharged to extended care facilities. J Am Coll Surg 1999;189(5):437-441.

18. Laupland KB, Kirkpatrick AW, Kortbeek JB, Zuege DJ. Long-term mortality outcome associated with prolonged admission to the ICU. Chest 2006;129(4):954-959.

19. Zilberberg MD, Luippold RS, Sulsky S, Shorr AF. Prolonged acute mechanical ventilation, hospital resource utilization, and mortality in the United States. Crit Care Med 2008;36(3):724-730.

20. Carson SS, Bach PB. Predicting mortality in patients suffering from prolonged critical illness: an assessment of four severity-of-illness measures. Chest 2001;120(3):928-933.

21. Zilberberg MD, de Wit M, Pirone JR, Shorr AF. Growth in adult prolonged acute mechanical ventilation: implications for healthcare delivery. Crit Care Med 2008;36(5):1451-1455.

22. Rapoport J, Teres D, Zhao Y, Lemeshow S. Length of stay data as a guide to hospital economic performance for ICU patients. Med Care 2003;41(3):386-397.

23. Kahn JM, Rubenfeld GD, Rohrbach J, Fuchs BD. Cost savings attributable to reductions in intensive care unit length of stay for mechanically ventilated patients. Med Care 2008;46(12):1226-1233.

24. Chelluri L, Mendelsohn AB, Belle SH, Rotondi AJ, Angus DC, Donahoe MP, et al. Hospital costs in patients receiving prolonged mechanical ventilation: does age have an impact? Crit Care Med 2003;31(6):1746-1751.
25. Gracey DR. The Problem With Diagnosis Related Group 475. Chest 2002;122(1):299-301.

26. Mayer-Oakes SA, Oye RK, Leake B, Brook RH. The early effect of Medicare's prospective payment system on the use of medical intensive care services in three community hospitals. JAMA 1988; 260(21):3146-3149.

27. Gracey DR, Hardy DC, Koenig GE. The chronic ventilator-dependent unit: a lower-cost alternative to intensive care. Mayo Clin Proc 2000;75(5):445-449.

28. Dasgupta A, Rice R, Mascha E, Litaker D, Stoller JK. Four-year experience with a unit for long-term ventilation (respiratory special care unit) at the Cleveland Clinic Foundation. Chest 1999;116(2): 447-455.

29. Quinnell TG, Pilsworth S, Shneerson JM, Smith IE. Prolonged invasive ventilation following acute ventilatory failure in COPD: weaning results, survival, and the role of noninvasive ventilation. Chest 2006;129(1):133-139.

30. Latriano B, McCauley P, Astiz ME, Greenbaum D, Rackow EC. Non-ICU Care of Hemodynamically Stable Mechanically Ventilated Patients. Chest 1996;109(6):1591-1596.

31. Douglas S, Daly B, Rudy E, Song R, Dyer MA, Montenegro H. The cost-effectiveness of a special care unit to care for the chronically critically ill. JONA: J Nurs Admin 1995;25(11):47-53.

32. Hoffman LA, Miller TH, Zullo TG, Donahoe MP. Comparison of 2 models for managing tracheotomized patients in a subacute medical intensive care unit. Respir Care 2006;51(11):1230-1236.

33. Solberg BCJ, Dirksen CD, Nieman FHM, van Merode G. Changes in hospital costs after introducing an intermediate care unit: a comparative observational study. Crit Care 2008;12(3):R68.

34. Polverino E, Nava S, Ferrer M, Ceriana P, Clini E, Spada E, et al. Patients' characterization, hospital course and clinical outcomes in five Italian respiratory intensive care units. Intensive Care Med 2010; 36(1):137-142.

35. Lilly CM, Zuckerman IH, Badawi O, Riker R. Benchmark data from greater than 240,000 adults that reflect the current practice of critical care in the United States. Chest 2011;140(5):1232-1242.

36. Kandilov AMG, Dalton K. Utilization and payment effects of Medicare referrals to long term care hospitals (LTCHs). Final Report June 2011. http://www.rti.org/reports/cms/HHSM-500-2006-000081/PDF/ CMS_LTCH_Referral_Effects.pdf.

37. Sebelius K. CMS Report: Determining medical necessity and appropriateness of care for medicare long term care hospitals. Department of Health and Human Services. 2011 Sep. 1;1-128. https://www. cms.gov/OfficeofLegislation/downloads/RTC-Long-Term-CareHospitals-Final.pdf.

38. Munoz Price LS. Long-term acute care hospitals. Clin Infect Dis 2009;49(3):438-443.

39. Program for Evaluating Payment Patterns Electronic Report. Top 20 DRGs for long-term acute care hospitals. April 2011. http://www. pepperresources.org/LinkClick.aspx ?fileticket $=$ YqVc78uBNb0\%3d \& tabid $=61$

40. Medicare Payment Advisory Commission (MEDPAC). Report to the Congress: new approaches in Medicare, Chapter 5: Defining longterm care hospitals. 2004;1-18. http://www.medpac.gov/publications/ congressional_reports/June04_ch5.pdf.

41. Scheinhorn DJ, Hassenpflug MS, Votto JJ, Chao DC, Epstein SK, Doig GS, et al. Post-ICU mechanical ventilation at 23 long-term care hospitals: a multicenter outcomes study. Chest 2007;131(1):85-93.

42. Lindsay ME, Bijwadia JS, Schauer WW, Rozich JD. Shifting care of chronic ventilator-dependent patients from the intensive care unit to the nursing home. Jt Comm J Qual Saf 2004;30(5):257-265.

43. Grabowski DC. Recent Trends In State Nursing Home Payment Policies. Health Aff (Millwood) 2004;(Suppl):W4-363-373. 
44. Mor V, Intrator O, Feng Z, Grabowski DC. The revolving door of rehospitalization from skilled nursing facilities. Health Aff (Millwood) 2010;29(1):57-64.

45. Unroe M, Kahn JM, Carson SS, Govert JA, Martinu T, Sathy SJ, et al. One-year trajectories of care and resource utilization for recipients of prolonged mechanical ventilation: a cohort study. Ann Intern Med 2010;153(3):167-175.
46. American Thoracic Society. Understanding costs and cost-effectiveness in critical care report from the second American Thoracic Society Workshop on Outcomes Research. Am J Respir Crit Care Med 2002;165(4):540-550.

47. Cox CE, Carson SS, Govert JA, Chelluri L, Sanders GD. An economic evaluation of prolonged mechanical ventilation. Crit Care Med 2007;35(8):1918-1927.

\section{Discussion}

Muldoon: ${ }^{*}$ I think the questions you raise on the artificiality of the whole venue system are valid, but the question that it begs is why this tiny percentage of acute care discharges that make up the CCI cost so much. We don't really know what to do with them, who should do it, or, to some degree, where it should be done, because there are so many options. We've got several choices: one is to leave it the way it is and muddle through, because it's only half a percent of discharges and not all that much money when you think about the big health care picture. Or we can bite it off and say that this is a group that's particularly vulnerable, confusing, and costly, and try to figure out whether this CCI belongs in a continued care hospital pilot or part of a risk-sharing entity where the numbers are large enough to make that work. Third, we can suffer through the regulatory management that we've had over the last 10 years. I'm open to answers.

MacIntyre: Shannon, I'm going to put you on the spot here. We've had some conversations on the topic, and you've been very good to try and educate me on these problems. You made a comment during one of these conversations that the short-term acute care hospital doesn't have the mindset or the culture to handle these long-

\footnotetext{
* Sean R Muldoon MD MPH, Kindred Healthcare, Hospital Division, Louisville, Kentucky.
}

term patients as well as do specialized centers. The short-term acute care hospitals have the facilities, the space, the nurses, and the staff, but not the mindset of taking care of a long-term patient. Did I phrase that properly?

Carson: Yes, the typical ICU in an acute hospital does not focus on these patients in a way that's always best for the patient. They're the last patients they get to on rounds each morning, and the physicians and nurses are frustrated. There are often tensions between caregivers and the families of these patients. They don't push rehab the way that they should, they're still throwing 3 antibiotics at every low-grade temperature, and ordering a CT for something that was scanned 3 weeks ago, because they lost track. So it's good to get these patients out of the ICU and into the SCU, within or outside the hospital.

That's what prompted these models: physicians back in the 1980s figured out that a different environment is necessary for these patients. And that's been a benefit. It's not widely implemented, but where it has I think it's been of benefit. Mike Donahoe did a really good job of making it clear that it's not the whole answer, because of artificial payment structures: a lot of patients reach the limit of availability of the resource while the need still exists.

When I rounded at an LTAC hospital, we were constantly trying to wean patients and implement rehab. All too often, however, patients would meet the end of the criteria to stay in the LTAC before our goals were achieved. We had to discharge them and we knew they were not going to survive outside the LTAC environment, without acute care. So that's the problem. What do you do with those patients?

White: About 5 years ago one of the clinical leaders at the Tufts Medical Center, where I was working in the ICU before I took the job at the LTAC, said something very prescient: he said that the acute hospitals have figured out how to manage their length of stay by using the LTACs. Now the problem is that the LTACs have to figure out a way to solve the same problem, and we are looking towards SNFs.

Bertuola: $\dagger$ Having had vast experience with the SNF and CCI patients, sometimes they're not critically ill when they come to us. All stable ventilator patients are in a fragile state. Sometimes the patients go back to the hospital because the stability is fragile, just like in the ICU. You get them stable one day, the next day they're unstable due to an infection. They're just so chronically ill with so many comorbidities, and yet we have patients in our centers who stay there for years and never bounce back to the hospital. You have this big mix of patients, and I think the SNFs can and do a good job at taking care of some of these patients and looking at dif-

\footnotetext{
$\dagger$ Lorraine Bertuola RRT, Genesis Healthcare, Kennett Square, Pennsylvania.
} 
ferent aspects of their care, talking about their activities of daily living and rehab, and really pushing that hard.

In our centers we wean a lot of patients; they've had a lot of chances to wean, but sometimes it's more mental than physical. In the hospital they come in to put the patient on a weaning trial and then leave, and the patient's anxiety is not well managed, leaving the patient very frightened. We get that type of patient weaned off the ventilator, though it may take as long as 3 or 4 months. We wean about $12 \%$ of our patients after they came to the SNF. So weaning is happening: we're seeing success.

But our focus is different; our focus starts with looking at the patient's quality of life. What can they do? Can we get them to the shower? Can we get them involved with activities with their families and rehab? I just want to point out that there is a time and a place for a patient to be in an SNF.

Nelson: I just wanted to comment that we've been referring to a population of patients who can't live outside an ICU. And many of the LTACs and SCUs have become ICUs, though they do it with somewhat less staffing. If you try to send chronically critically ill patients into any lower level of care, they will either die or bounce back to a higher level of care. The question really becomes, then what? Will we continue to support these patients, knowing that they really cannot exist for any sustained period of time without intensive care in some environment? That's a major societal question. What will we do with them? Will we maintain them indefinitely with intensive care? It has taken a long time for us to face that issue.

One of the consequences of discharging to LTACs has been that patients are then "lost to follow-up": the ICU clinicians don't see what happens later. Now people are coming to terms with this vast population, looking past discharge from the acute hospital, facing the longer-term outcomes, and what they see is that many of the patients with chronic critical illness just can't survive without continuing intensive care.

Snyder: $\div$ I think you made a great point, in that the tangible data we have is muddled by the varying aspects of LTACs and what is classified as an LTAC. Some are psychiatric hospitals, and some others are taking care of the sickest of the sick. I think it does muddle the data as far as staffing models and even mortality and some other statistics in the literature. I think it makes it difficult for people who are there every day to answer those questions we're having to answer. You bring up a great point as far as the intangible: cardiovascular ICUs and surgical ICUs specialize in specific problems. The LTAC is more like a CCI ICU, in that they concentrate on those patients and work with them and hopefully know them a little better. The intangible is very difficult to get into the literature in that setting, as well as many other settings in our hospitals.

Cheifetz: Since I was invited as the token pediatrician, I'll offer a pediatric perspective. Listening to this discussion, I appreciate how complex this topic is in the adult population. There are clearly many options for adults among the various LTACs and SNFs. In pediatrics the issue of resource allocation is even more pertinent, as there are very few LTAC or SNF equivalents for infants and children. In all of North Carolina there are only 2 long-term care facilities that accept chronically ventilated pediatric patients, and only one that considers infants and small children. So we find that pediatric ICUs and step-down/ special care units frequently have several of these patients who can be mechanically ventilated for months, and

\$ Lisa Snyder MD MPH, Select Medical, Mechanicsburg, Pennsylvania. sometimes up to a year, because we have no alternative facilities that will accept them in transfer. In terms of finances and resource allocation, this approach is clearly not optimal, but we often have no other option.

Muldoon: At Kindred Healthcare we've considered pediatrics and have, in fact, put a toe in the water with a couple of hospitals. Our ability to do that was hampered by 2 factors: one is that it never hit critical mass. There were 3 patients in one hospital and 2 in another, but you need a pediatric staff and pediatric specialists or you run the risk of treating them like little adults, which we know not to do. The second factor is that pediatric patients are there for the long haul. The mechanically ventilated pediatric patient is not a 2-month admission or even a 2-year admission. One of our patients had 15 birthdays with us.

Cheifetz: I completely appreciate the limitations. Thus, we often find ourselves transitioning these chronically ventilated infants and children from the acute care setting to their home rather than a LTAC. This approach, of course, raises a whole other set of hurdles and difficulties, which we will discuss tomorrow.

MacIntyre: Mike, I'm struck again by definitions. But it's also interesting that respiratory only account for about a third of the patients in the LTACs. Who are the other two thirds? Are their outcomes the same, or different?

Donahoe: I don't think in the public domain the outcome data are as well known for the non-ventilated patients, because they are not as easily classified.

Muldoon: There's a way to do it. The most current MedPAR file is 2 years old, so we just got 2009. So these are probably 2008 data, but for the whole industry the percentage of LTAC admissions who end up with 
a ventilator-related DRG is about $27 \%$. Now, the ventilator days are closer to $50 \%$, simply because stay is so much longer. The MedPAR file could tell us the top DRGs, but in the top $10 \mathrm{I}$ think 6 of them look like respiratory disorders.

MacIntyre: But let's emphasize that CCI and PMV are overlapping syndromes. I'm just trying to get a handle on who these non-ventilated CCI patients are. Shannon, maybe you can help me here?

Carson: That's an extremely important question and CMS has been hung up on it for a while. They also want to know who these patients are. We had a couple of technical panels to try to define these patients, and it's difficult. And it sounds trite, but they're real. Some of them are complex wound care; some are patients who are critically ill and have survived critical illness and are off the ventilator but otherwise they look just like the ventilated patients. They are atrophied and delirious and they need a lot of nursing and respiratory care. Some of them have tracheostomies and need tracheostomy care.

To me they look like CCI patients without a ventilator. They don't move them too far down the hall, because they could easily get back on the ventilator again. So I think CMS is uptight about who these patients are and why they need nursing care for so long. But they do, and I think it's been a big challenge to define them on paper. We know them when we see them, but on paper is different. There are some groups working on it, but I don't know how successful they've been so far at defining them in a clean way.

Snyder: The other category or DRG you would find is sepsis, as another portion of that two thirds.

Cabrera-Cancio: I agree with some of the prior comments, but would also like to address some issues pertaining to the amount of antimicrobial agents used in this population, as well as the number of tests and procedures these patients undergo. There are many settings where CCI patients receive care. The LTAC hospitals have a high percent of these patients and offer a unique setting to become familiar with their needs. Doing less may be better for these patients. Any small clinical change should not trigger a multitude of new orders, tests, new medications, et cetera.

An example: a new fever. It may be an infection, but it may also be caused by a non-infectious process. Not every fever requires multiple wide spectrum and expensive antibiotics. Knowing the patient and being cautious may be best. We should not underestimate the importance of having a dedicated, multidisciplinary team that knows the individual patients well.

In these long-term care facilities the number of physicians and the number of ancillary service healthcare workers is limited. The patients stay for prolonged periods; they are the focus of attention. In contrast, these patients in the acute care setting would likely receive less attention, since they will suffer the stigma of "not being as interesting," and healthcare workers frequently become frustrated by the lack of perceived progress.
In the acute care setting, physicians rotate daily and frequently, shifts occur, and continuity is lacking. Their focus, understandably is acute care and immediate, aggressive action.

Donahoe: I agree completely. If you've ever tried to set up a step-down unit and structure and staff it with nurses willing to work in that environment, and then recruit therapists to work with chronically critically ill patients, it's very difficult. I view this as a potential partnership that should evolve between academic medical centers, which carry the heaviest burden of these patients by far, and the LTAC industry.

The question is how the science of CCI disease management will advance. You can think of LVRS [lung volume reduction surgery] as a possible example to follow. This was an operation that was very expensive, and people were dying with unclear selection criteria for surgery. Medicare created a combined research/clinical model to study the indications for the disease. They essentially said, you can have the surgery if you agree to be randomized, and we'll pay for it and collect the data. Now the right patients get LVRS and the wrong people don't.

It's probably the only time something like that has been done in the history of medicine, and you have to wonder whether that model of clini$\mathrm{cal} /$ research design should be repeated for the CCI population. We should be able to study the patients across the continuum without artificial restrictions, so that as a clinician I would know better when to make my transfer decisions and when to have the appropriate end-of-life discussions.

This article is approved for Continuing Respiratory Care Education credit. For information and to obtain your CRCE

(free to AARC members) visit

www.RCJournal.com 